\title{
Hurricane Eyewall Evolution in a Forced Shallow-Water Model
}

\author{
ERIC A. HENDRICKS \\ Marine Meteorology Division, Naval Research Laboratory, Monterey, California \\ WAYNE H. SCHUBERT \\ Colorado State University, Fort Collins, Colorado \\ Yu-HAN CHEN AND Hung-CHI KuO \\ National Taiwan University, Taipei, Taiwan \\ Melinda S. PENG \\ Marine Meteorology Division, Naval Research Laboratory, Monterey, California
}

(Manuscript received 27 September 2013, in final form 23 December 2013)

\begin{abstract}
A forced shallow-water model is used to understand the role of diabatic and frictional effects in the generation, maintenance, and breakdown of the hurricane eyewall potential vorticity (PV) ring. Diabatic heating is parameterized as an annular mass sink of variable width and magnitude, and the nonlinear evolution of tropical storm-like vortices is examined under this forcing. Diabatic heating produces a strengthening and thinning PV ring in time due to the combined effects of the mass sink and radial PV advection by the induced divergent circulation. If the forcing makes the ring thin enough, then it can become dynamically unstable and break down into polygonal asymmetries or mesovortices. The onset of barotropic instability is marked by simultaneous drops in both the maximum instantaneous velocity and minimum pressure, consistent with unforced studies. However, in a sensitivity test where the heating is proportional to the relative vorticity, universal intensification occurs during barotropic instability, consistent with a recent observational study. Friction is shown to help stabilize the PV ring by reducing the eyewall PV and the unstable-mode barotropic growth rate. The radial location and structure of the heating is shown to be of critical importance for intensity variability. While it is well known that it is critical to heat in the inertially stable region inside the radius of maximum winds to spin up the hurricane vortex, these results demonstrate the additional importance of having the net heating as close as possible to the center of the storm, partially explaining why tropical cyclones with very small eyes can rapidly intensify to high peak intensities.
\end{abstract}

\section{Introduction}

While it is well known that environmental, oceanic, and internal processes are all important, the underlying dynamics of tropical cyclone intensity variability is not well understood. It has been shown that internal dynamical processes can be very important for intensity and structural variability. Examples of these processes are vortex Rossby wave propagation and wave-mean flow interaction (Guinn and Schubert 1993; Montgomery and

Corresponding author address: Eric A. Hendricks, Naval Research Laboratory, 7 Grace Hopper Ave., Monterey, CA 93943. E-mail: eric.hendricks@nrlmry.navy.mil
Kallenbach 1997; Möller and Montgomery 1999), generation and evolution of vortical hot towers (Hendricks et al. 2004; Montgomery et al. 2006; Nguyen et al. 2008), eyewall replacement cycles (Willoughby et al. 1982), and barotropic instability of the eyewall and eyeeyewall mixing (Schubert et al. 1999).

The instability of the eyewall, its breakdown, and subsequent potential vorticity mixing between the eyewall and eye has been examined extensively in idealized numerical modeling frameworks that are unforced (adiabatic and quasi inviscid; Schubert et al. 1999; Kossin and Schubert 2001; Nolan and Montgomery 2000, 2002; Terwey and Montgomery 2002; Rozoff et al. 2006; Kwon and Frank 2005; Hendricks et al. 2009; Hendricks and 
Schubert 2010; Menelaou et al. 2013a). These studies provided a basic understanding of barotropic instability and vorticity mixing in the inner core, but a lack of diabatic effects was these studies' primary limitation. On the other end of the spectrum, full-physics modeling studies have also been conducted examining eyewall evolution with the inclusion of diabatic and frictional effects (Chen and Yau 2001; Yau et al. 2004; Kwon and Frank 2008; Yang et al. 2007; Wu et al. 2009; Nguyen et al. 2011; Menelaou et al. 2013b). Wu et al. (2009) examined the eyewall evolution for a landfalling typhoon, and investigated the roles of condensational heating and friction on the eyewall evolution. They found a complicated interaction between the friction and heating, whereby friction helps to keep the PV annulus narrow by the stretching deformation during the heating. Nguyen et al. (2011) documented vacillation cycles of symmetric-to-asymmetric (and vice versa) transitions in a simulation of Hurricane Katrina (2005), and examined the structural and intensity variability associated with these transitions. While these studies are more realistic, it is more difficult to understand the salient dynamics because of the complexity of these simulations. In addition to the full-physics modeling studies, observational and experimental studies have also been conducted (Montgomery et al. 2002; Reasor et al. 2000; Kossin and Eastin 2001; Kossin et al. 2002; Kossin and Schubert 2004; Hendricks et al. 2012). The observational studies highlight the complex nature of eyewall mesovortices and eye-eyewall mixing in the real atmosphere.

The study of Rozoff et al. (2009) provided a bridge of the gap between the idealized unforced studies and the forced full-physics modeling studies. They performed idealized experiments examining hurricane evolution in a forced nondivergent barotropic model, where diabatic heating was parameterized as a vorticity forcing term. Their main conclusion was that barotropic instability and subsequent vorticity mixing is a transient intensification brake during symmetric intensification; however, the transport of high-vorticity air into the eye from the eyewall eventually produces a higher-intensity vortex than one that did not undergo this internal mixing mechanism. This was the dual nature of potential vorticity mixing noted in their study. The Rozoff et al. (2009) study provided a simple framework to examine the dynamic instability mechanism in the presence of diabatic heating and friction.

The purpose of the present study is to extend the study of Rozoff et al. (2009) to the next level of complexity toward the real atmosphere by using a shallow-water model. The main difference between the forced nondivergent barotropic model and forced shallow-water model is the existence of divergence and inertia-gravity waves that can be excited by the heating in the latter model. In the shallow-water model used here, diabatic heating is parameterized as an annular mass sink, and the prototypical evolution of tropical storm-like vortices is examined. This work is intended to help bridge the gap between the idealized unforced studies and the fullphysics studies, allowing for a more complete understanding of evolution of the eyewall, and eyewall instability, in the presence of diabatic and frictional effects. Two important questions that we seek to answer are as follows: 1) How does diabatic heating modulate the potential vorticity field during axisymmetric intensification? and 2) What is the role of barotropic instability in the vortex intensity and structural variability in this forced framework? The rest of this paper is organized as follows. In section 2, the forced divergent barotropic equations and numerical discretizations are given. The initial conditions and forcing functions are described in section 3. The results of several control experiments are given in section 4, along with a wavemean flow diagnosis of the results. Sensitivity tests are given in section 5 . The conclusions are given in section 6 .

\section{Dynamical model}

\section{a. Governing equations}

The model is based upon the forced dissipative divergent barotropic (shallow water) equations on an $f$ plane. The governing equations are

$$
\begin{array}{r}
\frac{\partial u}{\partial t}+u \frac{\partial u}{\partial x}+v \frac{\partial u}{\partial y}-f v+g \frac{\partial h}{\partial x}=-\mu u+\nu \nabla^{2} u, \\
\frac{\partial v}{\partial t}+u \frac{\partial v}{\partial x}+v \frac{\partial v}{\partial y}+f u+g \frac{\partial h}{\partial y}=-\mu v+\nu \nabla^{2} v, \\
\frac{\partial h}{\partial t}+\frac{\partial(u h)}{\partial x}+\frac{\partial(v h)}{\partial y}=-h Q+\nu \nabla^{2} h,
\end{array}
$$

where $u$ is the zonal velocity, $v$ is the meridional velocity, $h$ is the fluid depth, $Q$ is the mass sink, $\mu$ is the coefficient of Rayleigh friction, $\nu$ is the diffusivity, and $f$ is the Coriolis parameter. The numerical model is based upon the vorticity divergence formulation of (1) and (2):

$$
\begin{aligned}
& \frac{\partial \zeta}{\partial t}+ \frac{\partial[(f+\zeta) u]}{\partial x}+\frac{\partial[(f+\zeta) v]}{\partial y}=-\mu \zeta+\nu \nabla^{2} \zeta, \\
& \frac{\partial \delta}{\partial t}-\frac{\partial[(f+\zeta) v]}{\partial x}+\frac{\partial[(f+\zeta) u]}{\partial y} \\
&+\nabla^{2}\left[g h+\frac{1}{2}\left(u^{2}+v^{2}\right)\right]=-\mu \delta+\nu \nabla^{2} \delta,
\end{aligned}
$$


where $\zeta=\partial v / \partial x-\partial u / \partial y$ is the relative vorticity and $\delta=$ $\partial u / \partial x+\partial v / \partial y$ is the divergence. While the focus of this work is on the nonlinear model above, it is useful to note that linearized versions of (3)-(5) about a circular vortex support inertia-gravity, vortex Rossby, and mixed gravity-Rossby waves (Montgomery and Lu 1997).

\section{b. Potential vorticity principle}

By combining (4) and (3), while eliminating the divergence, we obtain the potential vorticity principle

$$
\frac{D P}{D t}=P Q-\frac{\mu \zeta}{h}+\frac{\nu}{h}\left(\nabla^{2} \zeta-P \nabla^{2} h\right)
$$

where $P=(f+\zeta) / h$ is the potential vorticity, and $D / D t=$ $(\partial / \partial t)+u(\partial / \partial x)+v(\partial / \partial y)$ is the material derivative. Equation (6) indicates that $P$ is not materially conserved because of the mass sink term $P Q$, the Rayleigh friction term $\mu \zeta / h$, and the diffusion term. While the numerical model solves (3)-(5), the PV equation in (6) will be used to interpret the dynamics.

\section{c. Discretization}

Numerical simulations were performed using a pseudospectral code based on (3)-(5). The numerical solution was obtained on a $600 \mathrm{~km} \times 600 \mathrm{~km}$ doubly periodic domain using $512 \times 512$ equally spaced collocation points. After dealiasing of the quadratic advection terms in (3)-(5), the number of retained Fourier modes was $170 \times 170$, yielding a wavelength of the highest Fourier mode of $3.53 \mathrm{~km}$. A third-order Adams-Bashforth time scheme was used with a time step of $5 \mathrm{~s}$. Ordinary diffusion is used to help control nonlinear instability, and the coefficient was set to $\nu=25 \mathrm{~m}^{2} \mathrm{~s}^{-1}$. The Coriolis parameter was set to $f=3.77 \times 10^{-5} \mathrm{~s}^{-1}$ and the mean fluid depth was $\bar{h}=4077 \mathrm{~m}$, supporting inertia-gravity wave phase speeds of $200 \mathrm{~m} \mathrm{~s}^{-1}$.

\section{Initial condition and heating function}

\section{a. Initial condition}

The initial condition is a tropical storm-like vortex. The tropical storm vortex is constructed as a slightly smoothed Rankine vortex according to

$$
\bar{\zeta}(r, 0)= \begin{cases}\zeta_{1} & 0 \leq r \leq r_{3}, \\ \zeta_{1} S\left(\frac{r-r_{3}}{r_{4}-r_{3}}\right)+\zeta_{2} S\left(\frac{r_{4}-r}{r_{4}-r_{3}}\right) & r_{3} \leq r \leq r_{4}, \\ \zeta_{2} & r_{4} \leq r<\infty,\end{cases}
$$

where $\zeta_{1}=1 \times 10^{-3} \mathrm{~s}^{-1}, \zeta_{2}, r_{3}=58 \mathrm{~km}$, and $r_{4}=62 \mathrm{~km}$ are constants, and $S(s)=1-3 s^{2}+2 s^{3}$ is a cubic Hermite

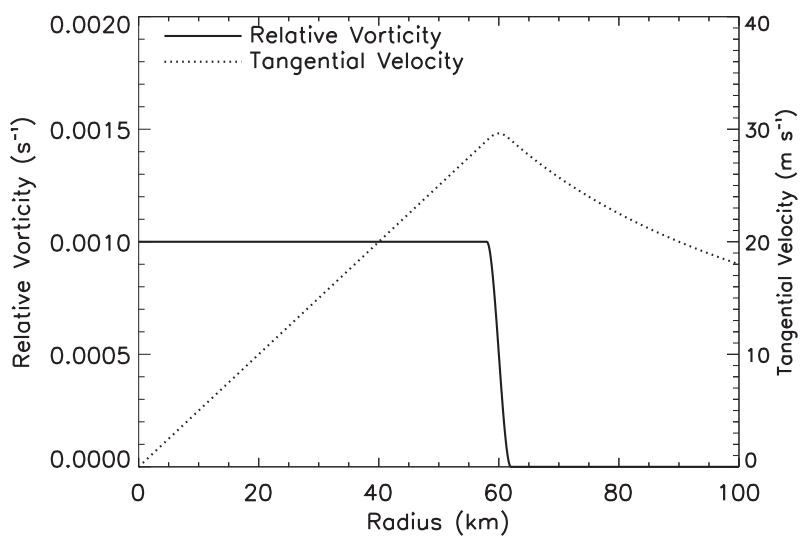

FIG. 1. Initial condition for the shallow-water model control experiments. The relative vorticity and tangential velocity are shown for the tropical storm-like vortex.

shape function that provides smooth transition zones. Here, $\zeta_{2}$ is set to a small negative value so that the domain-average vorticity vanishes. Plots of the initial vorticity and winds are given in Fig. 1 . Note that the peak winds at $r=60 \mathrm{~km}$ are approximately $30 \mathrm{~m} \mathrm{~s}^{-1}$.

\section{b. Heating function}

To parameterize the effects of eyewall diabatic heating in a shallow-water model, an axisymmetric, annular mass sink is specified with the following form:

$$
Q(r, t)=Q_{0} L(r) \alpha t e^{-\alpha t},
$$

where $Q_{0}$ and $\alpha$ are constants, and the spatial structure is given by

$$
L(r)= \begin{cases}0, & 0 \leq r \leq r_{1}, \\ S\left(\frac{r_{2}-r}{r_{2}-r_{1}}\right) & r_{1} \leq r \leq r_{2}, \\ 1, & r_{2} \leq r \leq r_{3}, \\ S\left(\frac{r-r_{3}}{r_{4}-r_{3}}\right) & r_{3} \leq r \leq r_{4}, \\ 0, & r_{4} \leq r \leq \infty,\end{cases}
$$

where $r$ denotes the radius; $r_{1}, r_{2}, r_{3}, r_{4}$ are the radii defining the annulus; and $S(s)$ is the same shape function used in (7). To specify the constant $Q_{0}$, an analogy is made to the isentropic coordinate version of the continuously stratified primitive equations, for which the continuity equation is

$$
\frac{\partial \sigma}{\partial t}+\frac{\partial(u \sigma)}{\partial x}+\frac{\partial(v \sigma)}{\partial y}=-\frac{\partial(\sigma \dot{\theta})}{\partial \theta}
$$




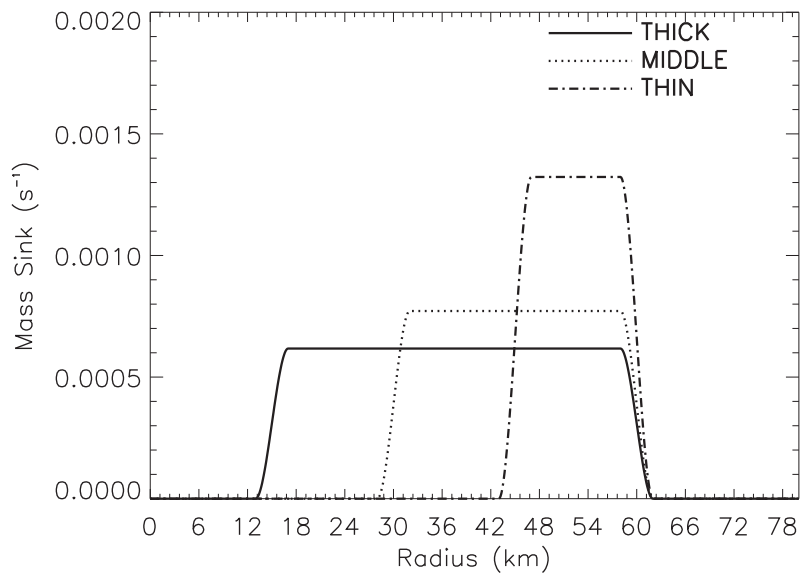

FIG. 2. Mass-sink profiles for the thick, middle, and thin heating regions with $\alpha^{-1}=1.0 \times 10^{4} \mathrm{~s}$. Each profile has the same areaintegrated heating.

where $\sigma=-(1 / g)(\partial p / \partial \theta)$ is the pseudodensity and $\dot{\theta}$ is the diabatic heating rate. When $\sigma$ varies slowly in the vertical, the right-hand side of (10) can be approximated by $-\sigma(\partial \dot{\theta} / \partial \theta)$, so that, in the analogy between (3) and (10), the shallow-water depth $h(x, y, t)$ is analogous to the pseudodensity $\sigma(x, y, \theta, t)$ and the shallow-water mass sink $Q(x, y, t)$ is analogous to $\partial \dot{\theta}(x, y, \theta, t) / \partial \theta$. The analogy can be sharpened by considering a two-layer version of the $\theta$-coordinate model, with the lower-tropospheric layer defined by $300<\theta<330 \mathrm{~K}$, with the maximum diabatic heating $\dot{\theta}_{m}(r, t)$ occurring at the midtropospheric level $\theta=$ $330 \mathrm{~K}$, and with negligible diabatic heating at the surface (Schumacher et al. 2004). Then, in the lower troposphere, $\partial \dot{\theta} / \partial \theta$ can be approximated by $\dot{\theta}_{m}(r, t) /(30 \mathrm{~K})$ and the analogy yields

$$
\dot{\theta}_{m}(r, t)=(30 \mathrm{~K}) Q_{0} L(r) \alpha t e^{-\alpha t} .
$$

The integral of (11) over area and time is

$$
\int_{0}^{\infty} \int_{0}^{\infty} \dot{\theta}_{m}(r, t) r d r d t=\frac{(30 \mathrm{~K}) Q_{0}\left(r_{b}^{2}-r_{a}^{2}\right)}{\alpha},
$$

where $r_{a}=\left(r_{1}+r_{2}\right) / 2, r_{b}=\left(r_{3}+r_{4}\right) / 2$, and the area integral has been approximated by considering the transition zones in (9) to be very narrow. To make the right-hand side of (12) independent of the choices for $\alpha$, $r_{a}$, and $r_{b}$, we have chosen $Q_{0}$ as

$$
Q_{0}=\frac{(50 \mathrm{~km})^{2}(250 \mathrm{~K}) \alpha}{\left(r_{b}^{2}-r_{a}^{2}\right)(30 \mathrm{~K})},
$$

and the constants $(50 \mathrm{~km})^{2}$ and $(250 \mathrm{~K})$ have been chosen for consistency with the satellite-derived, inner-core latent

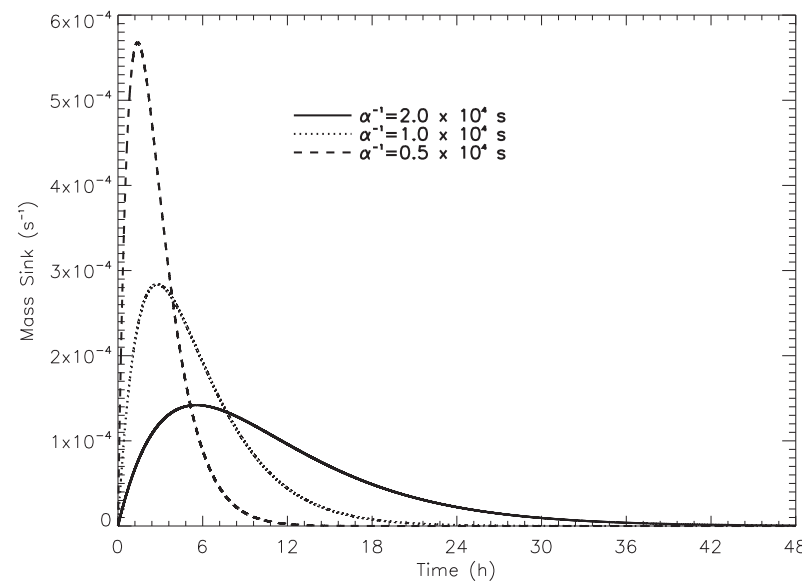

FIG. 3. Time variation of the heating function with different values of $\alpha$ for the thick heating annulus $\left(r_{1}, r_{2}, r_{3}, r_{4}\right)=(28,32$, $58,62) \mathrm{km}$.

heating rates found by Rodgers et al. (1998). To summarize, the use of (13) in (8) provides an annular forcing $Q(r, t)$, which has an area and time integral that is independent of the parameters $\alpha, r_{a}$, and $r_{b}$.

For the control experiments, three different size rings were chosen: (i) thick: $\left(r_{1}, r_{2}, r_{3}, r_{4}\right)=(13,17,58,62) \mathrm{km}$, (ii) middle: $\left(r_{1}, r_{2}, r_{3}, r_{4}\right)=(28,32,58,62) \mathrm{km}$, and (iii) thin: $\left(r_{1}, r_{2}, r_{3}, r_{4}\right)=(43,47,58,62) \mathrm{km}$. The radial structure of each mass sink $\left[Q_{0} L(r)\right]$ is shown in Fig. 2. For the temporal dependence, three different $\alpha$ values were chosen: (i) slow forcing: $\alpha^{-1}=2.0 \times 10^{4} \mathrm{~s}$, (ii) medium forcing: $\alpha^{-1}=1.0 \times 10^{4} \mathrm{~s}$, and (iii) fast forcing: $\alpha^{-1}=0.5 \times 10^{4} \mathrm{~s}$. The temporal dependence of the mass sink $\left[Q_{0} \alpha t \exp (-\alpha t)\right]$ for the thick heating annulus is given in Fig. 3 .

\section{Control simulations}

\section{a. Results}

Nine control simulations were run, as listed in Table 1. These control simulations span the matrix defined by three $\alpha$ values (slow to fast forcing) and three annulus widths (thick to thin). The qualitative results of these experiments are described here. The control experiments are run without Rayleigh friction $(\mu=0)$; the sensitivity to friction will be examined later.

Before examining all of the control experiments, details of the experiment A1 (thick heating with slow

TABLE 1. Matrix of control experiments.

\begin{tabular}{lccc}
\hline & $\alpha^{-1}=2.0 \times 10^{4} \mathrm{~s}$ & $\alpha^{-1}=1.0 \times 10^{4} \mathrm{~s}$ & $\alpha^{-1}=0.5 \times 10^{4} \mathrm{~s}$ \\
\hline Thick & A1 & A2 & A3 \\
Middle & B1 & B2 & B3 \\
Thin & C1 & C2 & C3
\end{tabular}




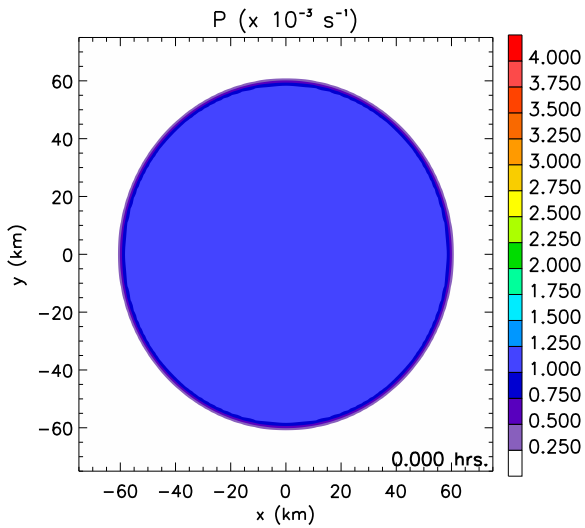

c)

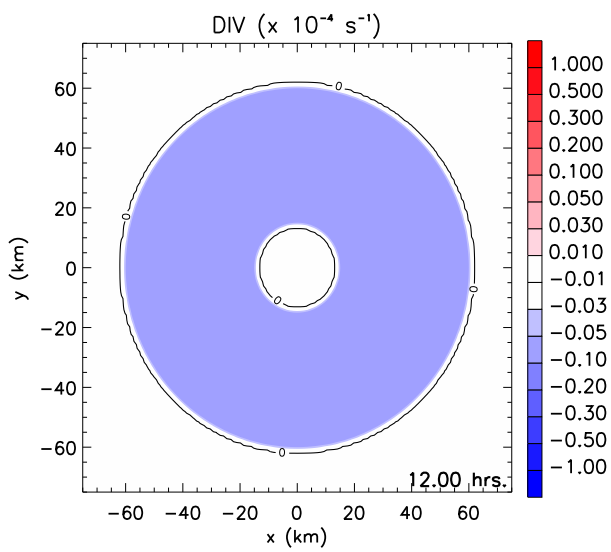

b)

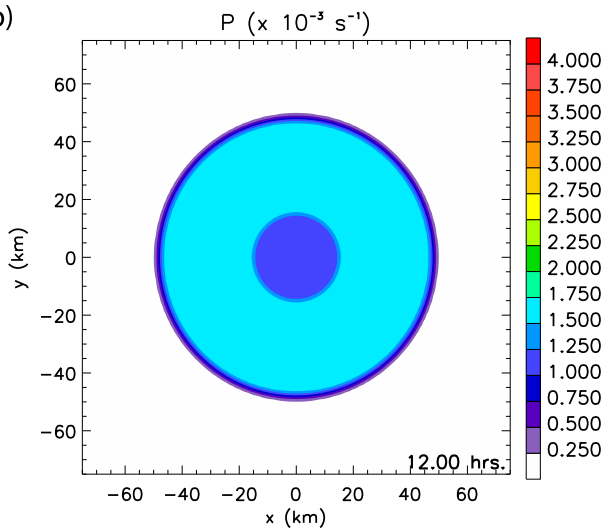

d)

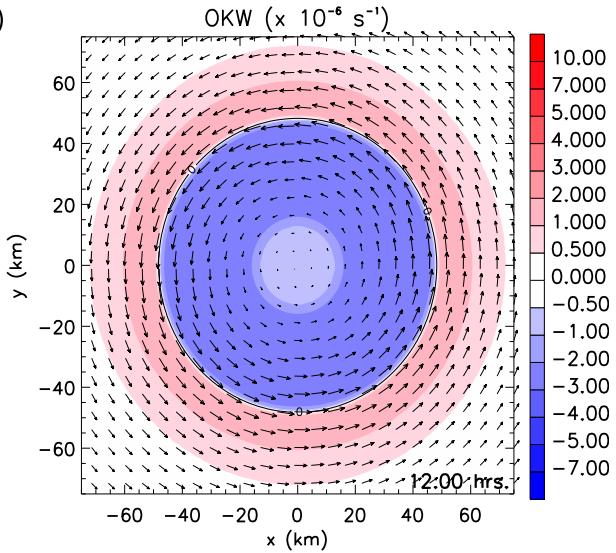

FIG. 4. Evolution of experiment A1. (a) Potential vorticity at $t=0 \mathrm{~h}$, (b) potential vorticity at $t=12 \mathrm{~h}$, (c) divergence at $t=12 \mathrm{~h}$, and (d) Okubo-Weiss parameter at $t=12 \mathrm{~h}$ with flow vectors overlaid.

forcing) are given in Fig. 4. In Fig. 4a, the PV at $t=0$ is shown, exhibiting a Rankine-like initial condition. Figure $4 \mathrm{~b}$ shows the PV at $t=12 \mathrm{~h}$, which exhibits a ringlike structure in response to the mass sink, which produces a thick annular region of convergence (Fig. 4c). As the outer radius of the PV ring shrinks, the Okubo-Weiss parameter $\left(\mathrm{OKW}=s_{n}^{2}+s_{s}^{2}-\zeta^{2}\right.$, where $s_{n}=\partial u / \partial x-$ $\partial v / \partial y$ is the stretching deformation and $s_{s}=\partial v / \partial x+\partial u / \partial y$ is the shearing deformation) is positive outside the radius of maximum wind and negative inside. During the simulation, the high-deformation region becomes larger as the radius of maximum wind shrinks in response to the contracting outer radius of the PV ring.

The results for all of the thick forcing experiments A1-A3 are given in Fig. 5. In each simulation, a PV ring develops in response to the thick mass sink. The PV increases most rapidly in A3, followed by $\mathrm{A} 2$ and then A1, as expected from the temporal forcing curves (Fig. $3)$. In each case, the ring remains stable at $t=48 \mathrm{~h}$. This is because the thick forcing produces a thick PV ring, which according to the linear stability analysis of Schubert et al. (1999) is more likely to remain stable. All three cases are similar at $t=48 \mathrm{~h}$ since the spatial and time-integrated heating is the same.

The results for middle forcing experiments B1-B3 are given in Fig. 6, with the initial condition being the same as in Fig. 5. For the slow temporal forcing experiment $\mathrm{B} 1$, at $t=6 \mathrm{~h}$, a very weak PV ring has developed, which intensifies by $t=24 \mathrm{~h}$, and at $t=48 \mathrm{~h}$, a faint azimuthal wavenumber-5 pattern is distinguishable as the ring is just becoming unstable. For the faster forcing experiments B2 and B3, the rings becomes unstable by $t=24 \mathrm{~h}$, resulting in wavenumber-4 polygonal eyewall structures. The instability proceeds more rapidly for the fast forcing experiment B3. At $t=48 \mathrm{~h}$, both experiments have polygonal eyewall shapes. In the context of the Schubert et al. (1999) linear stability analysis, the PV ring for case B2 at $t=24 \mathrm{~h}$ would be most unstable to azimuthal wavenumber 5 (using PV as a proxy for vorticity). Therefore, it is possible that the square domain and grid structure is favoring wavenumber-4 perturbations, helping to excite a wavenumber-4 breakdown. However, the Schubert et al. (1999) linear stability analysis was done in the context of the linearized nondivergent 


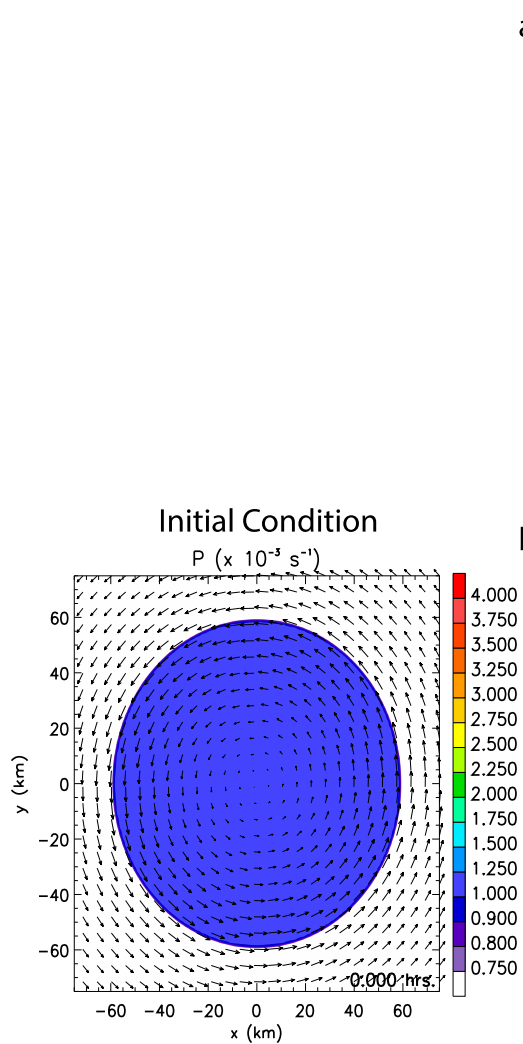

a) $\mathrm{A} 1$

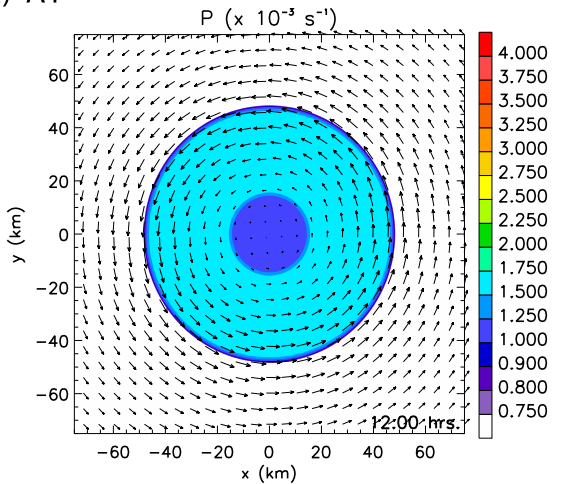

b) $\mathrm{A} 2$

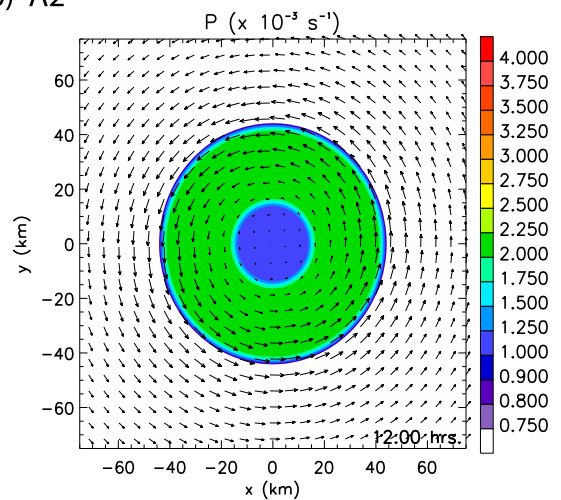

c) $\mathrm{A3}$

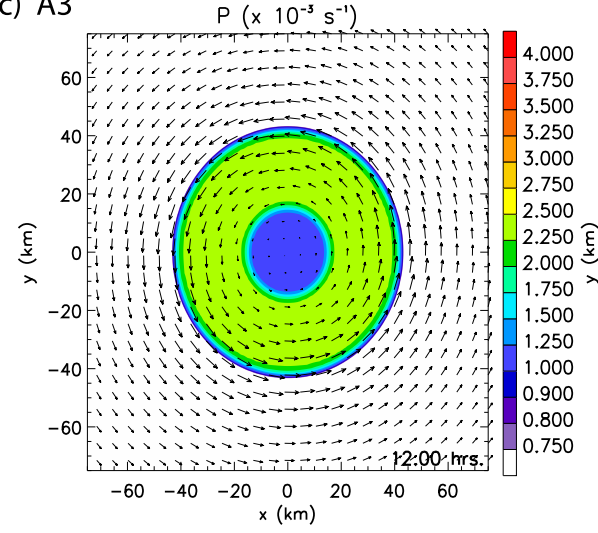

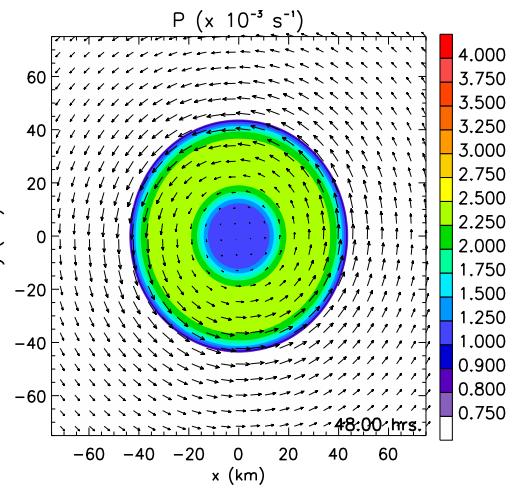
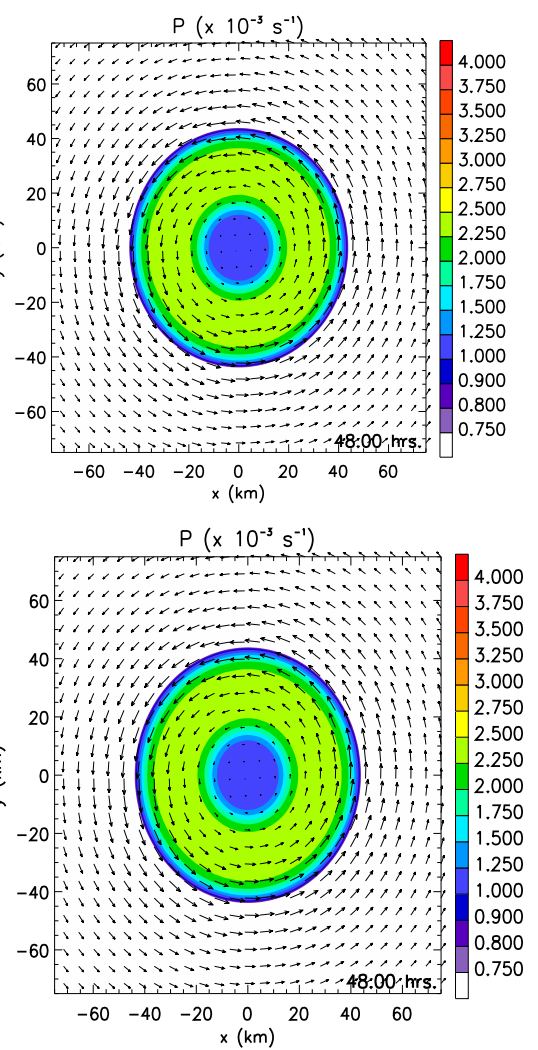

FIG. 5. Evolution of the thick ring for different heating rates. (a) A1: $\alpha^{-1}=2.0 \times 10^{4} \mathrm{~s}$, (b) A2: $\alpha^{-1}=1.0 \times 10^{4} \mathrm{~s}$, and (c) A3: $\alpha^{-1}=0.5 \times 10^{4} \mathrm{~s}$. (left) $t=0$, (middle) $t=12$, and (right) $t=48 \mathrm{~h}$. Wind vectors are overlaid in each panel.

barotropic vorticity equation, not the shallow-water equations, and therefore caution should be taken in direct comparisons. Qualitatively, the Schubert et al. (1999) results are a good guide to the evolution of these rings in that thinner PV rings break down at higher azimuthal wavenumbers and at faster rates.

The results for the thin forcing experiments $\mathrm{C} 1-\mathrm{C} 3$ are given in Fig. 7. Here, all experiments result in thin rings that break down into multiple mesovortices initially, from which mergers and interactions occur resulting in fewer mesovortices. For the slow forcing experiment $\mathrm{C} 1$, a thin eyewall PV ring develops at $t=6 \mathrm{~h}$. At $t=13.5 \mathrm{~h}$, this ring becomes unstable and a wavenumber- 12 pattern is evident. Then the ring breaks down into mesovortices, and mergers occur, resulting in a pattern of eight mesovortices at $t=48 \mathrm{~h}$. For the medium forcing experiment $\mathrm{C} 2$, the evolution is similar but proceeds more rapidly. The breakdown of the ring happens earlier, resulting in 12 mesovortices at $t=13.5 \mathrm{~h}$. Mergers occur with this case as well, resulting in eight mesovortices at $t=48 \mathrm{~h}$. In the fast forcing experiment $\mathrm{C} 3$, the PV becomes unstable by $t=6 \mathrm{~h}$, resulting in the ring 
a) B1

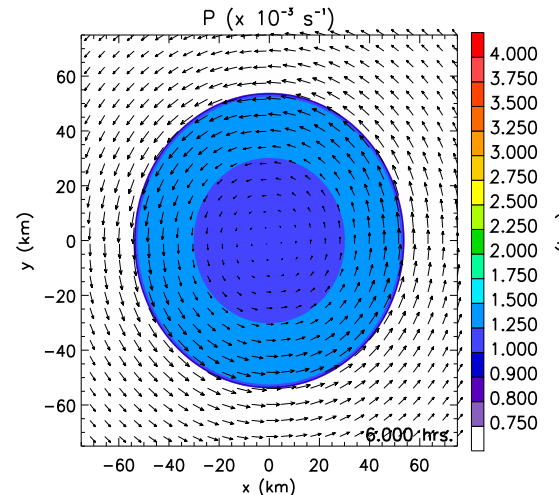

b) B2

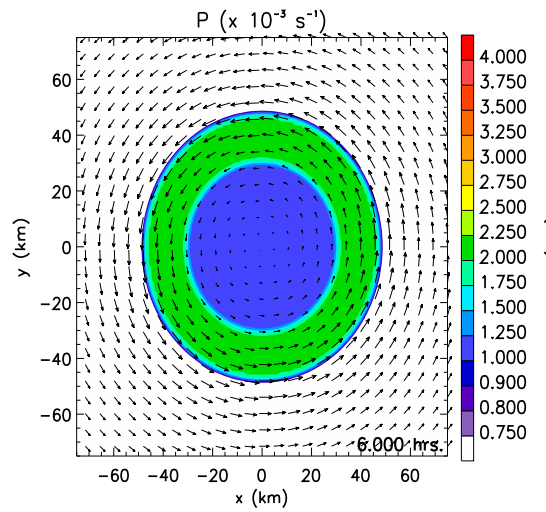

c) B3

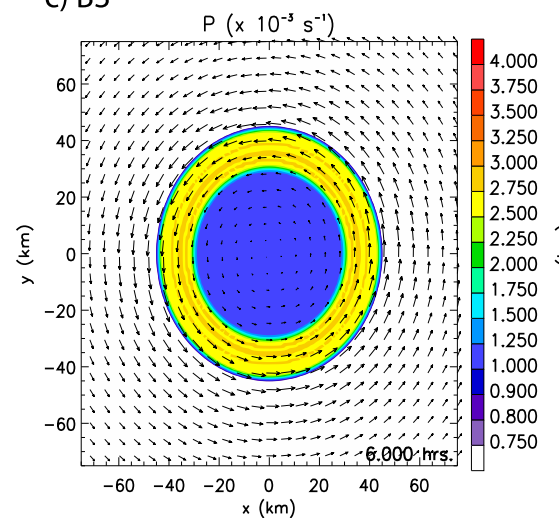

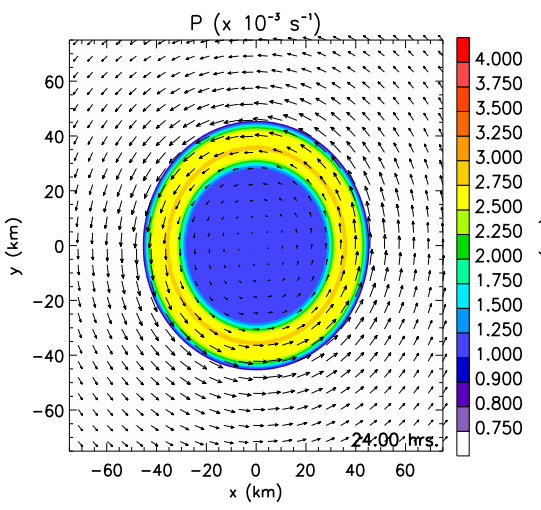
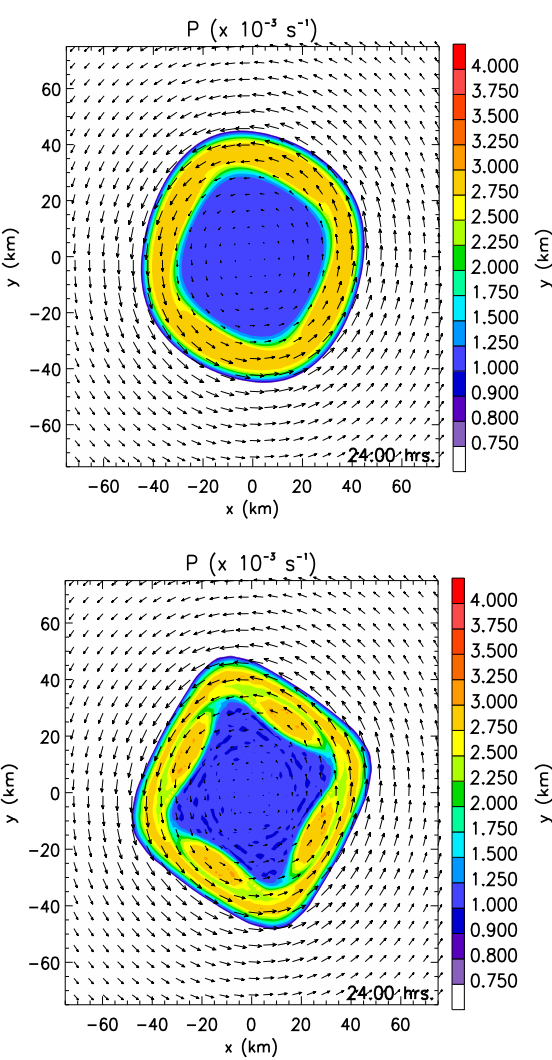
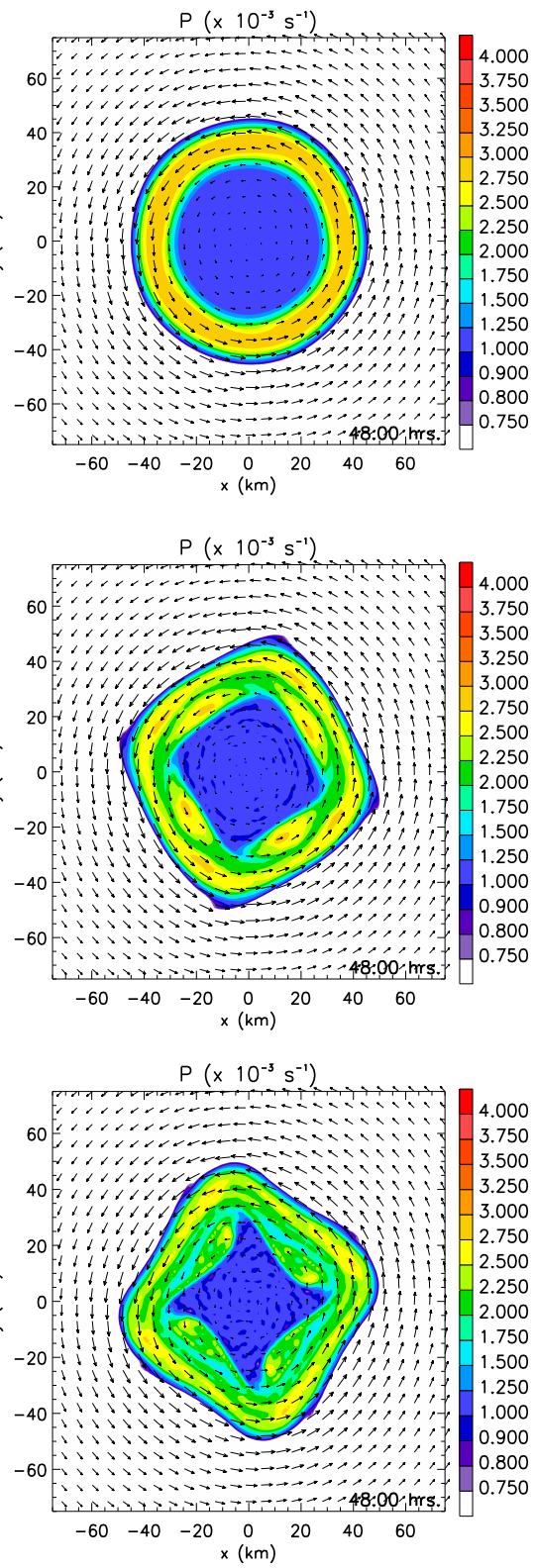

FIG. 6. Evolution of the middle ring for different heating rates. (a) B1: $\alpha^{-1}=2.0 \times 10^{4} \mathrm{~s}$, (b) B2: $\alpha^{-1}=1.0 \times 10^{4} \mathrm{~s}$, and (c) B3: $\alpha^{-1}=0.5 \times$ $10^{4} \mathrm{~s}$. (left) $t=6$, (middle) $t=24$, and (right) $t=48 \mathrm{~h}$. Wind vectors are overlaid in each panel.

breakdown and a pattern of four mesovortices at $t=$ $48 \mathrm{~h}$. These three simulations were also integrated out to $120 \mathrm{~h}$ to examine more details of the evolution of the mesovortices. In experiment $\mathrm{C} 1$, a monopole formed at $t=61 \mathrm{~h}$. In experiments $\mathrm{C} 2$ and $\mathrm{C} 3$, monopoles formed at approximately $t=110 \mathrm{~h}$, as four long-lived mesovortices merged. These results indicate that after the heating shuts off, the nonlinear evolution of the mesovortices (including vortex mergers into a monopole) is different for each experiment.
It is insightful to understand the evolution of the forced rings using the potential vorticity equation in (6). Rewriting (6) in cylindrical coordinates, and neglecting the friction and diffusion terms, we obtain

$$
\frac{\partial P}{\partial t}=-v_{r} \frac{\partial P}{\partial r}-v_{\phi} \frac{\partial P}{r \partial \phi}+P Q
$$

where $v_{r}$ is the radial velocity, $v_{\phi}$ is the tangential velocity, $r$ is the radius from the origin, and $\phi$ is the azimuthal 
a) $\mathrm{C} 1$
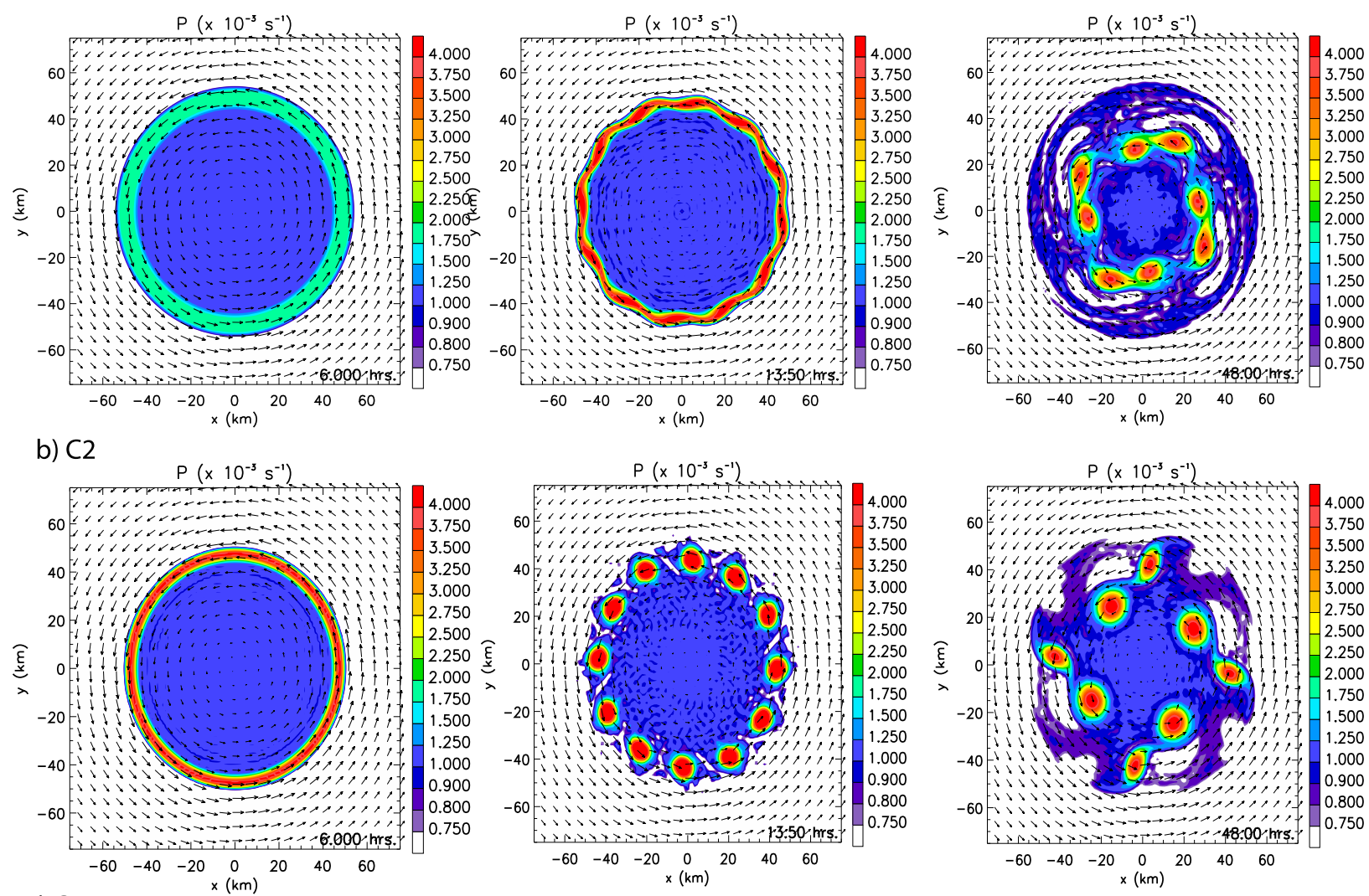

c) $\mathrm{C} 3$
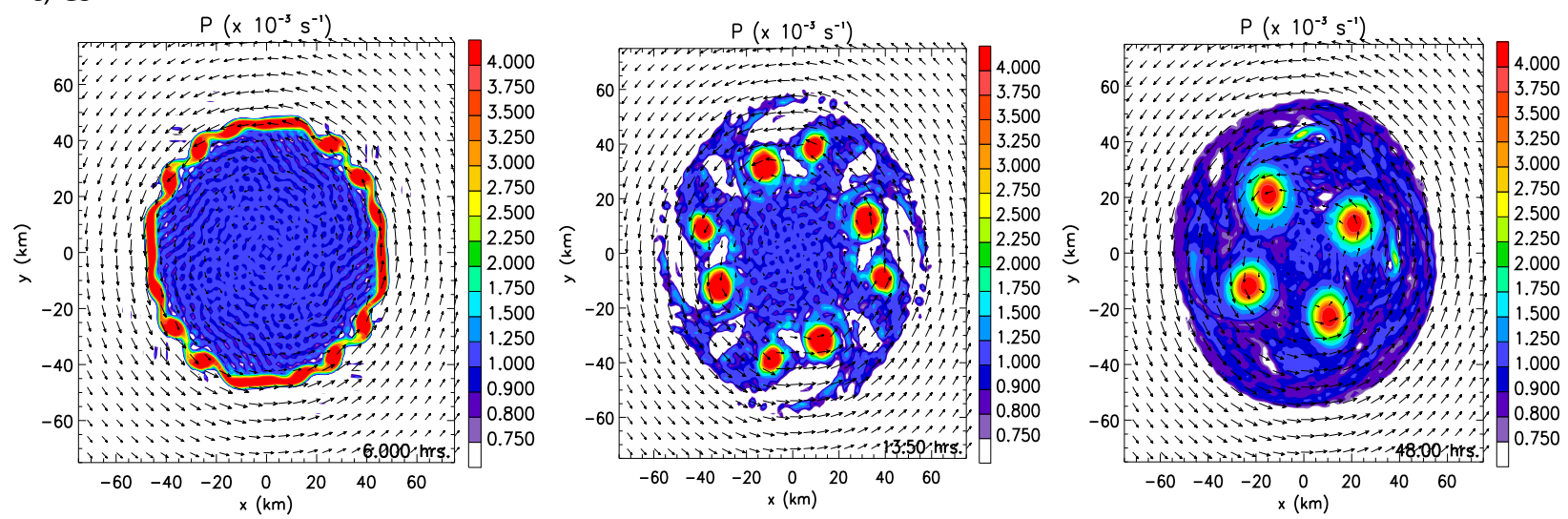

FIG. 7. Evolution of the thin ring for different heating rates. (a) $\mathrm{C} 1: \alpha^{-1}=2.0 \times 10^{4} \mathrm{~s}$, (b) $\mathrm{C} 2: \alpha^{-1}=1.0 \times 10^{4} \mathrm{~s}$, and (c) $\mathrm{C} 3: \alpha^{-1}=0.5 \times 10^{4} \mathrm{~s}$. (left) $t=6$, (middle) $t=13.5$, and (right) $t=48 \mathrm{~h}$. Wind vectors are overlaid in each panel.

angle. For forced rings that remain axisymmetric (barotropic instability does not set in), the second term on the right-hand side of (14) is zero and the local PV change is governed by the radial advection term $\left[-v_{r}(\partial P / \partial r)\right]$ and mass sink term $(P Q)$. In the region of the forcing, the mass sink term is continually producing PV. In each simulation, the PV rings become thinner in time, with the maximum $\mathrm{PV}$ production occurring near the inner radius of the heating annulus. This is due to the radial advection term. The heating produces a divergent circulation $v_{r}$ that advects lower-PV fluid inward, thus reducing PV at the outer edge of the heating annulus. The inward advection is favored in the outer region where the inertial stability is weaker, while the PV production is favored in the inner region where the PV is larger. Therefore, axisymmetric forcing produces a strengthening and thinning of the $\mathrm{PV}$ 


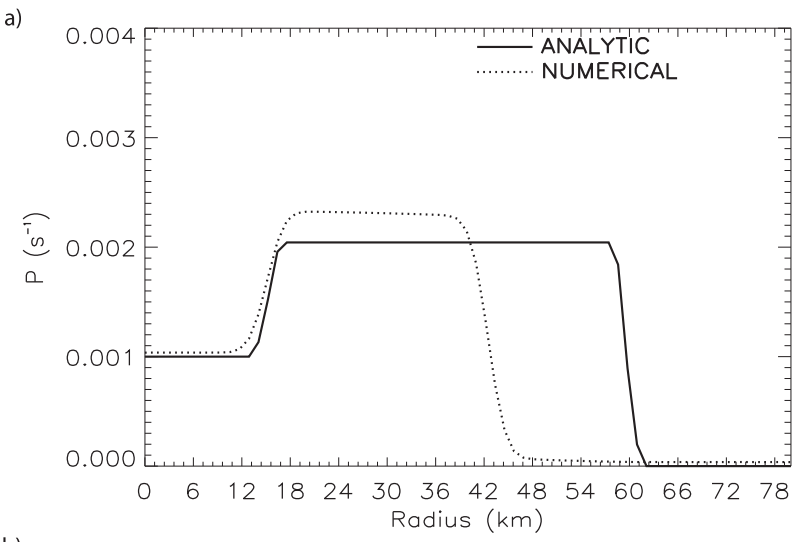

b)

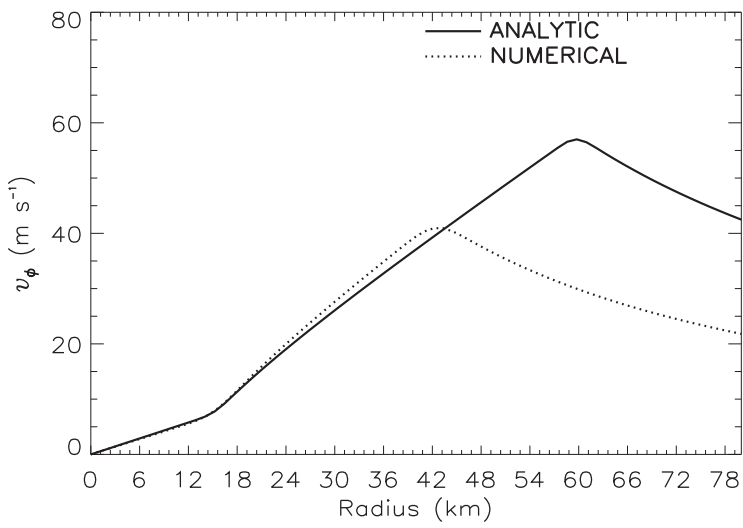

FIG. 8. (a) Comparison of analytic solution for the final potential vorticity to the azimuthal mean at $t=48 \mathrm{~h}$ from the numerical experiment (case A1). The analytic solution, given by $P(r, \infty)=$ $P(r, 0) \exp \left[Q_{0} L(r) / \alpha\right]$, does not include the effect of radial advection of PV. (b) Comparison of the corresponding profiles of the tangential velocity.

annulus in time, with a maximum value at the inner radius of the heating. The importance of this will be discussed in the next section, which examines intensity variability.

Mathematically, this can be further illustrated by neglecting the PV advection terms in (14), obtaining

$$
\frac{\partial P}{\partial t}=P Q
$$

which has the analytic solution $P(r, \infty)=P(r, 0) \exp$ $\left[Q_{0} L(r) / \alpha\right]$. Using the thick heating ring $\mathrm{A} 1$ as an example (since it remains stable in the numerical simulation), a direct comparison of the numerical and analytic solution of PV can be made for this case. The comparison of the final PV (i.e., the PV resulting from the net heating) is given in Fig. 8a. The analytic PV is given by the solid curve while the numerical PV solution (containing radial advection of PV) is given by the dotted curve. As shown here, the main difference between the numerical and analytic PV is that the numerical solution exhibits a thinner final PV ring with a slightly larger peak value. This clearly illustrates the role of the radial advection of $P V$ by the induced divergent circulation. The divergent circulation produces a thinning PV ring in time, as lower PV is advected inward. The induced radial $\mathrm{PV}$ advection also causes a reduction in the radius of maximum wind and a reduction in the magnitude of the maximum wind, as compared to the case with no radial PV advection. This is illustrated in Fig. 8b.

\section{b. Intensity variability}

In this section, the intensity variability is examined for two groups of experiments: the first group varies the heating rate ( $\alpha$ parameter), while the second group varies the thickness of the heating annulus. The results are shown in Fig. 9. In Figs. 9a-c, the heating rate is varied for the thick, middle, and thin heating regions, respectively. In Figs. 9d-f, the heating width is varied for the same heating rates. The maximum instantaneous velocity in the model domain is used as the intensity metric. In Fig. 9a, the maximum wind speeds for the thick rings are given for the varying heating rates. As expected, $\alpha^{-1}=0.5 \times 10^{4} \mathrm{~s}$ has the most rapid intensification rate. All simulations reach the same intensity at $t=48 \mathrm{~h}$ because the total heating is the same for each case. Moving to the middle heating annulus (Fig. 9b), similar results are seen in the early stage of the simulation, but since the $\alpha^{-1}=1.0 \times 10^{4}$ and $\alpha^{-1}=$ $0.5 \times 10^{4} \mathrm{~s}$ simulations become unstable (Fig. 5) in the later stage, some variability exists. In particular, both simulations have a slightly lower maximum wind speed at $t=48 \mathrm{~h}$ than the $\alpha^{-1}=2.0 \times 10^{4} \mathrm{~s}$ simulation due to the onset of barotropic instability. For the thin ring experiments (Fig. 9c), the $\alpha^{-1}=0.5 \times 10^{4}$ s experiments have the fastest intensification rates. At the onset of barotropic instability, in each case the maximum wind speed decreases. After the instability and during the PV mixing process, the fast heating rate experiment $\left(\alpha^{-1}=0.5 \times 10^{4} \mathrm{~s}\right)$ exhibits the lowest intensity by maximum wind.

In Fig. $9 \mathrm{~d}, \alpha^{-1}=2.0 \times 10^{4} \mathrm{~s}$ is held constant for varying heating annuli thicknesses. The thin heating annulus has the lowest intensity due to barotropic instability and the middle heating case has a lower intensity than the thick heating. Similar results are shown in Figs. 9e and 9f; however, in these cases the middle heating becomes slightly unstable, leading to a reduced intensity. It is interesting to note that the thick and middle heating experiments have different intensities at $t=48 \mathrm{~h}$, considering they have the same net heating. The reason for the higher intensity of the thick heating annulus is that it has a smaller inner radius. The significance of this will be further discussed in the next section. For rings that remain stable, the minimum central 
a)

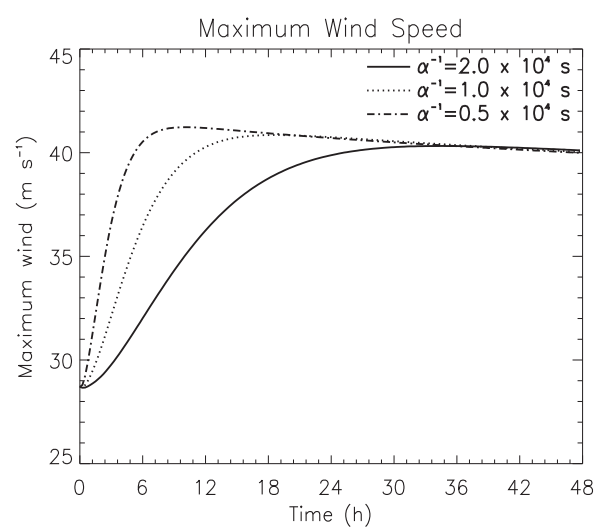

b)

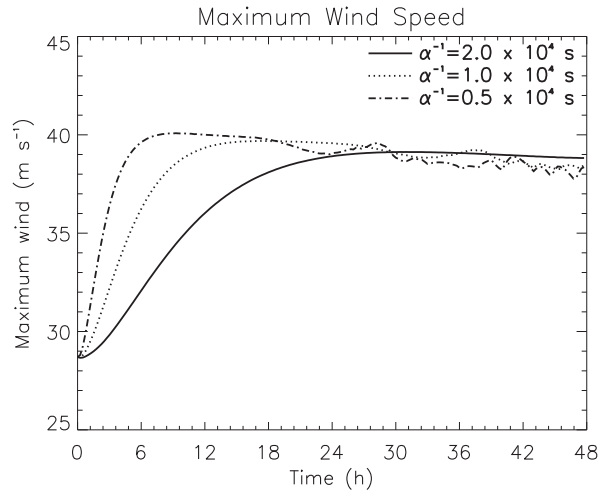

c)

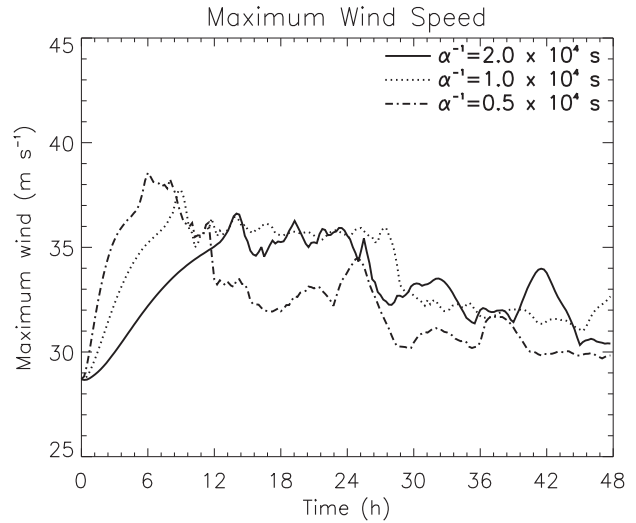

d)

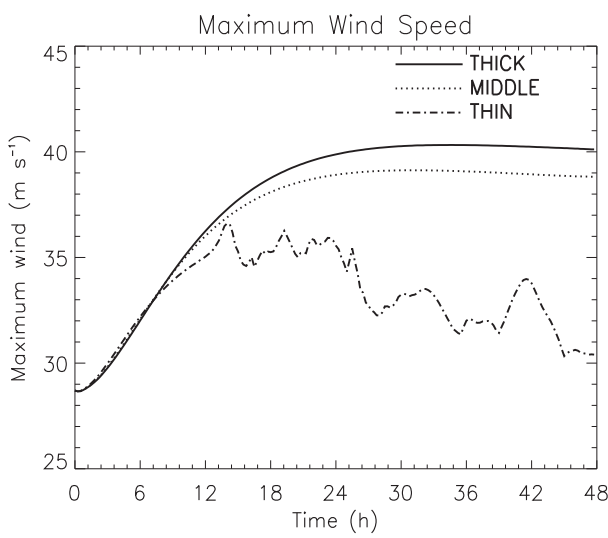

e)

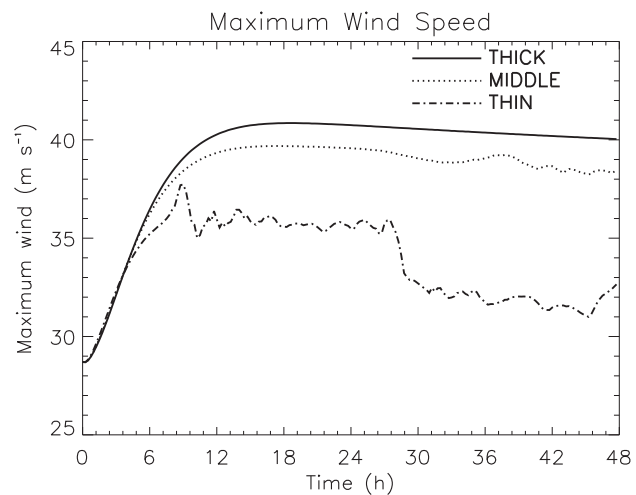

f)

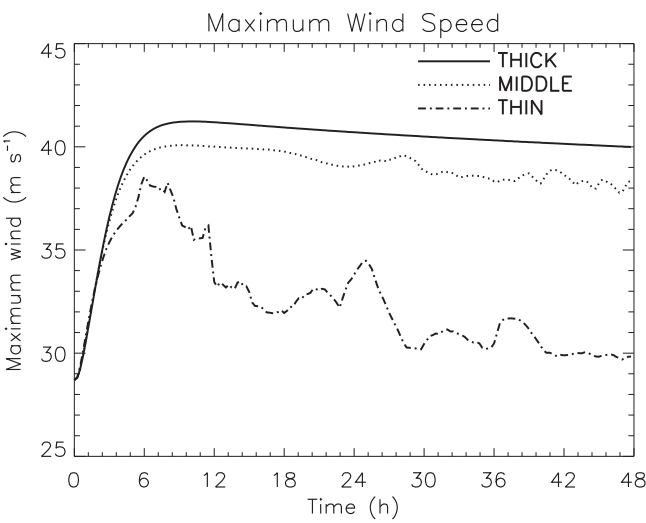

FIG. 9. Evolution of the maximum velocity as function of $\alpha$ and ring thickness. (a) Thick heating varying $\alpha$ (A1, A2, A3), (b) middle heating varying $\alpha$ (B1, B2, B3), and (c) thin heating varying $\alpha(\mathrm{C} 1, \mathrm{C} 2, \mathrm{C} 3)$; (d) $\alpha^{-1}=2.0 \times 10^{4} \mathrm{~s}$ with varying thickness (A1, B1, C1), (e) $\alpha^{-1}=1.0 \times 10^{4} \mathrm{~s}$ with varying thickness (A2, B2, C2), and (f) $\alpha^{-1}=0.5 \times 10^{4}$ s with varying thickness (A3, B3, C3). Note that $\alpha^{-1}=2.0 \times 10^{4}, 1.0 \times 10^{4}$, and $0.5 \times 10^{4} \mathrm{~s}$, for slow, middle, and fast heating, respectively.

pressure $\Delta p=\rho g \Delta h$ exhibits the same intensity behavior as the maximum velocity (i.e., the runs with rapid intensification and high peak intensities also have rapid deepening and low central pressures). For the rings that become unstable and break down, the minimum central pressure deepens while the maximum velocity decreases, consistent with prior unforced studies.
Synthesizing the above results, barotropic instability acts as a transient intensification brake during symmetric annular forcing, confirming the forced nondivergent barotropic model results of Rozoff et al. (2009). The results also confirm the nondivergent results of Schubert et al. (1999) and Kossin and Schubert (2001) with simultaneous reductions in maximum wind and minimum 
pressure during barotropic instability (not shown). These results also show that the variability in the intensity is sensitive to the heating rate, even long after the heating has ceased. The maximum wind at $t=48 \mathrm{~h}$ is substantially lower for those rings that became unstable versus rings that did not become unstable.

\section{c. Absolute angular and tangential momentum budgets}

To further understand the dynamics of the intensity variability, tangential momentum and absolute angular momentum budgets are performed. First, the polarcoordinate versions of (1)-(3) are used (neglecting nonconservative effects), where $r$ is the radius and $\phi$ is the azimuthal angle:

$$
\begin{gathered}
\frac{D v_{r}}{D t}-f v_{\phi}-\frac{v_{\phi}^{2}}{r}+g \frac{\partial h}{\partial r}=0, \\
\frac{D v_{\phi}}{D t}+f v_{r}+\frac{v_{r} v_{\phi}}{r}+g \frac{\partial h}{r \partial \phi}=0, \\
\frac{\partial h}{\partial t}+\frac{\partial\left(r h v_{r}\right)}{r \partial r}+\frac{\partial\left(h v_{\phi}\right)}{r \partial \phi}=0,
\end{gathered}
$$

where $v_{r}$ and $v_{\phi}$ are the radial and tangential velocities (as in section $4 \mathrm{a})$, and $D / D t=(\partial / \partial t)+v_{r}(\partial / \partial r)+\left(v_{\phi} / r\right)(\partial / \partial \phi)$ is the material derivative in polar coordinates. The wavemean flow interaction theory described here for the shallow-water equations is a special case of the isentropic coordinate formulation developed by Andrews (1983) and Tung (1986) and reviewed in the text by Andrews et al. (1987). For $v_{r}$, the mass-weighted azimuthal average is defined by $\hat{v}_{r}=\overline{h v_{r}} / \bar{h}$, where the ordinary azimuthal average is defined as

$$
\bar{v}_{r}(r)=\frac{1}{2 \pi} \int_{0}^{2 \pi} v_{r}(r, \lambda) d \phi .
$$

The deviation from the ordinary azimuthal average is defined by $v_{\phi}^{\prime}=v_{\phi}-\bar{v}_{\phi}$, and the deviation from the mass-weighted average is defined by $v_{r}^{*}=v_{r}-\hat{v}_{r}$. Similar definitions hold for the other variables.

Applying $\overline{()}$ to each term in (18) and noting that $\overline{h v_{r}}=\bar{h} \hat{v}_{r}$, we can write the azimuthal-mean mass continuity equation as

$$
\frac{\partial \bar{h}}{\partial t}+\frac{\partial\left(r \bar{h} \hat{v}_{r}\right)}{r \partial r}=0 .
$$

Similarly, applying $\overline{()}$ to each term in (17) and noting that $\overline{h P v_{r}}=\bar{h} \hat{P} \hat{\boldsymbol{v}}_{r}+\overline{h P^{*} v_{r}^{*}}$, we can write the azimuthal-mean tangential momentum per unit mass $\left(\bar{v}_{\phi}\right)$ equation as

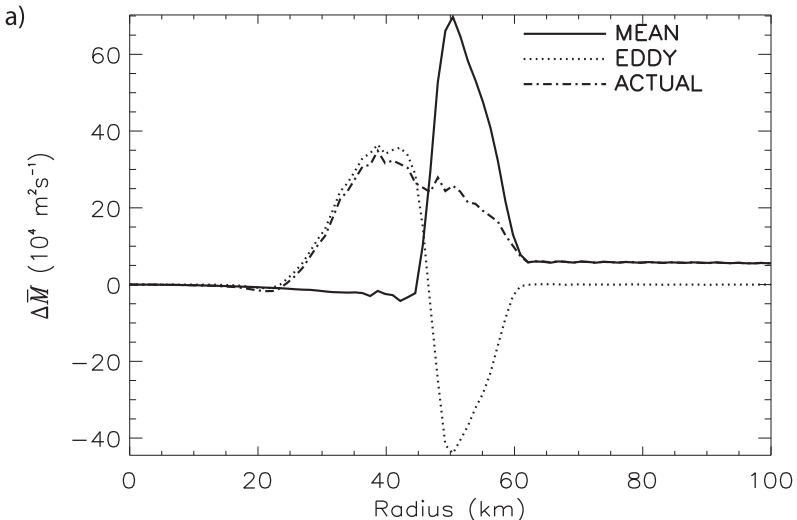

b)

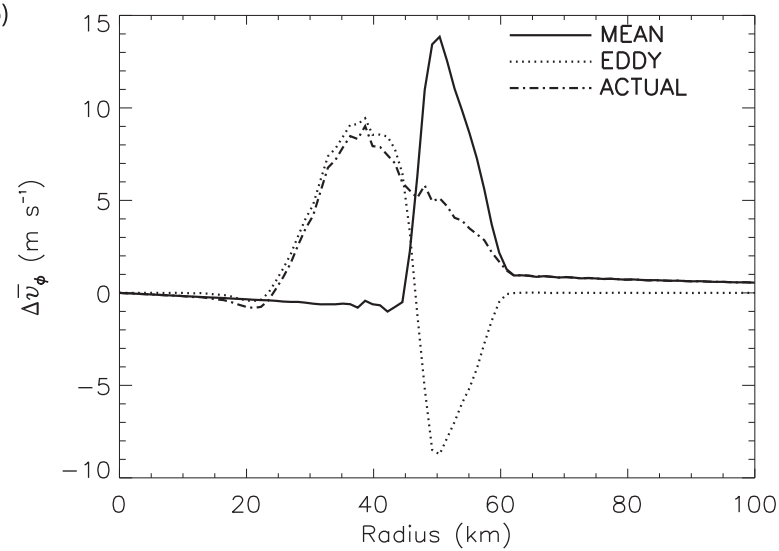

FIG. 10. Integrated 0-48-h (a) angular and (b) tangential momentum budgets for experiment $\mathrm{C} 1$.

$$
\frac{\partial \bar{v}_{\phi}}{\partial t}+(f+\bar{\zeta}) \hat{v}_{r}=-\overline{h P^{*} v_{r}^{*}} .
$$

This equation can be cast into the absolute angular momentum form,

$$
\frac{\partial \bar{M}}{\partial t}+\hat{v}_{r} \frac{\partial \bar{M}}{\partial r}=-r \overline{h P^{*} v_{r}^{*}},
$$

where $\bar{M}=r \bar{v}_{\phi}+(1 / 2) f r^{2}$ is the azimuthal-mean absolute angular momentum per unit mass.

To elucidate the roles of the eddy and mean processes, angular momentum budgets are performed for case $\mathrm{C} 1$ (thin ring with slow forcing). Recall that this ring had a mass sink in the region $45 \leq r \leq 60 \mathrm{~km}$. This ring broke down into mesovortices and is therefore a good case to examine the role of eddy processes. In Fig. 10, both the tangential momentum and absolute angular momentum budgets are shown. The "mean terms" are the second terms on the left-hand sides of (21) and (22), and the "eddy terms" are the terms on the right-hand sides of (21) and (22). To compute these terms, (21) and (22) are integrated with the trapezoidal rule over $48 \mathrm{~h}$ using the 10-min output data. The "actual terms" are the differences in the first term on the left-hand side of (21) and 
TABLE 2. List of sensitivity tests.

\begin{tabular}{cccl}
\hline \hline Experiment name & Annulus type & $\alpha^{-1}\left(\times 10^{4} \mathrm{~s}\right)$ & \multicolumn{1}{c}{ Description } \\
\hline C1X & Thin & 2.0 & C1 with Rayleigh friction \\
C1Y & Thin & 2.0 & C1 with increased diffusion \\
C1Z & Thin & 2.0 & C1 with 8-km transition zones \\
B2X & Middle & 1.0 & B2 with inner radius held fixed \\
C2X & Thin & 1.0 & C2 with inner radius held fixed \\
B2Y & Middle & 1.0 & B2 with heating centered on vortex edge \\
B2Z & Middle & 1.0 & B2 with heating outside vortex edge \\
A1X & Thick & 2.0 & A1 with $f$ at $5^{\circ} \mathrm{N}$ \\
A1Y & Thick & 2.0 & A1 with $f$ at $25^{\circ} \mathrm{N}$ \\
D1 & - & 2.0 & Heating proportional to vorticity \\
\hline
\end{tabular}

(22) from $t=0$ to $t=48 \mathrm{~h}$ (i.e., the temporal integral of the local tendency terms).

The axisymmetric heating has a dominant contribution to the mean term in the heating region $(45 \leq r \leq$ $60 \mathrm{~km}$ ). As the mass sink produces PV and converges vorticity, the circulation is increased in this region. Outside $r=50 \mathrm{~km}$, the increase is not as significant due to the axisymmetric inward radial advection of lowerPV fluid. Essentially, the vorticity is increased (decreased) in the inner (outer) region of the mass sink due to combination of the mass sink and radial PV advection. The eddy term is negative in the region $45 \leq r \leq$ $60 \mathrm{~km}$ and positive in the region $20 \leq r \leq 45 \mathrm{~km}$. As the $\mathrm{PV}$ ring breaks down during barotropic instability, $\mathrm{PV}$ is fluxed in an asymmetric manner from the eyewall to the eye. The eddy term shows how this PV mixing process affects the tangential and angular momentum budgets. As PV is mixed from the eyewall to the eye, the outer eye is spun up at the expense of the eyewall. Neglecting the minor effect of diffusion, the actual term is the summation of the mean and eddy contributions, and thus is positive in the entire outer-eye to eyewall region, $20 \leq r \leq 60 \mathrm{~km}$. Therefore, the net effect of the forced evolution and instability is to increase the tangential momentum in a large region in the inner core. It is also interesting to note that the induced divergent circulation increases the angular momentum for $r \geq 60 \mathrm{~km}$, as contours of absolute angular momentum are drawn inward.

\section{Sensitivity tests}

In addition to the control simulations, several sensitivity tests were conducted. These tests explore the sensitivity of the structural and intensity change to variations in forcing and initial parameters. A list of the sensitivity tests is given in Table 2, and they are described below.

\section{a. Inclusion of Rayleigh friction}

Since the control experiments did not have Rayleigh friction, a sensitivity test was conducted to understand how Rayleigh friction modifies the forced ring evolution (Table 2, experiments C1X). In Fig. 11, the thin heating ring experiment is run with $\alpha^{-1}=2.0 \times 10^{4} \mathrm{~s}$, both with and without Rayleigh friction (experiments $\mathrm{C} 1$ and C1X, respectively). The Rayleigh friction coefficient $\mu=4 \times 10^{-6} \mathrm{~s}^{-1}$ corresponds to a vorticity half-life of $48 \mathrm{~h}$, for which there is an observational basis in the atmosphere (Montgomery et al. 2001). As shown in Figs. 11a and 11b, Rayleigh friction helps stabilize the PV ring slightly during the eyewall development. According to (4) and (5), Rayleigh friction damps the vorticity and divergence with a proportional magnitude and also damps the PV according to (6). Friction will damp the vorticity in both the eyewall (mass sink) and eye regions, and friction will damp the convergence (induced by the mass sink) in the eyewall region. The slight decrease in eyewall vorticity reduces the radial shear of the tangential velocity and therefore would reduce the most unstable mode barotropic growth rate. The reduction in the convergence in the mass-sink region would slightly reduce the PV generation in that region. The combination of these processes has a stabilizing effect on the PV ring. These forced shallow-water model simulation results contradict the forced nondivergent barotropic model results of Rozoff et al. (2009), who found that Rayleigh friction causes slightly more unstable conditions by also reducing the vorticity in the eye region. While the eye vorticity is also reduced by friction in our simulations (apparent in Fig. 11b), the frictional modification of the eyewall processes is more important in these simulations. These results are similar to Rozoff et al. (2009) in that the 48-h half-life Rayleigh friction only slightly modifies the evolution of forced PV rings. Some differences between the present study and Rozoff et al. (2009) are due to the different models and heating parameterizations employed. In Rozoff et al. (2009), diabatic heating was parameterized as a logistically limited vorticity forcing term. As Rayleigh friction damps the vorticity in their simulations, it also modifies the logistic term. In this study, diabatic heating is 
a) NO FRICTION

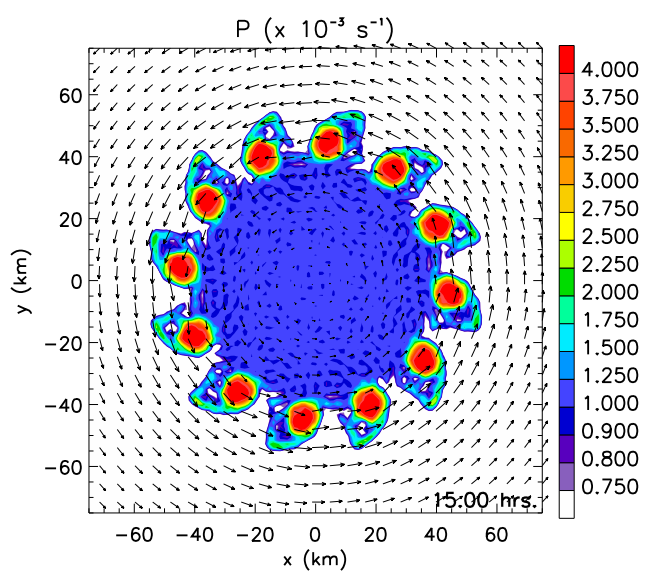

c) NO FRICTION

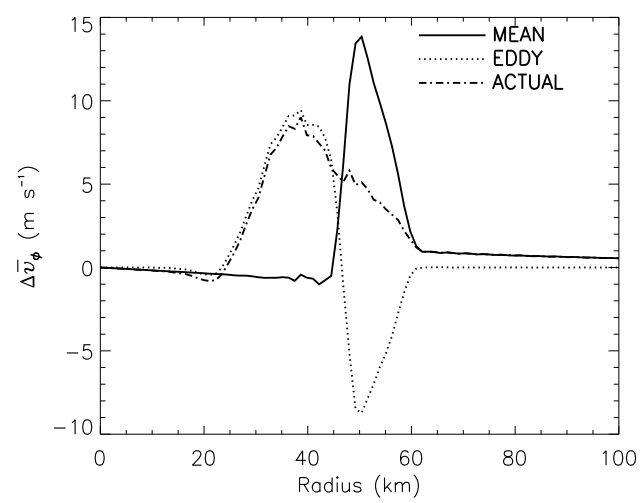

b) WITH FRICTION

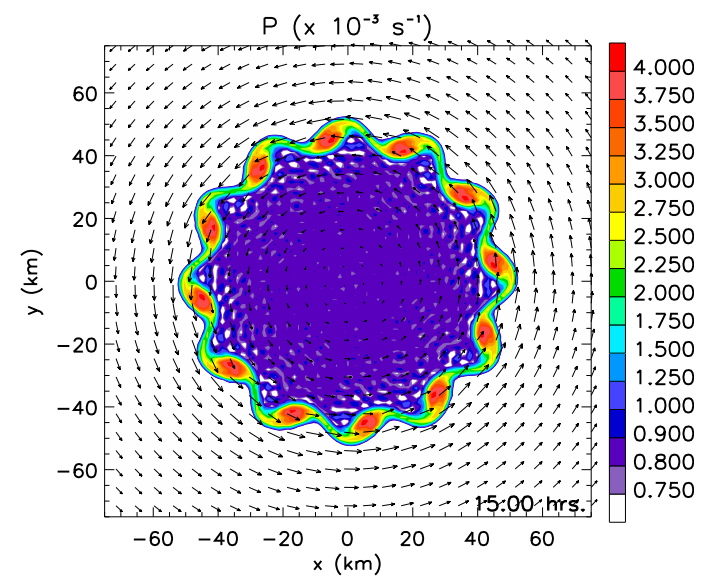

d) WITH FRICTION

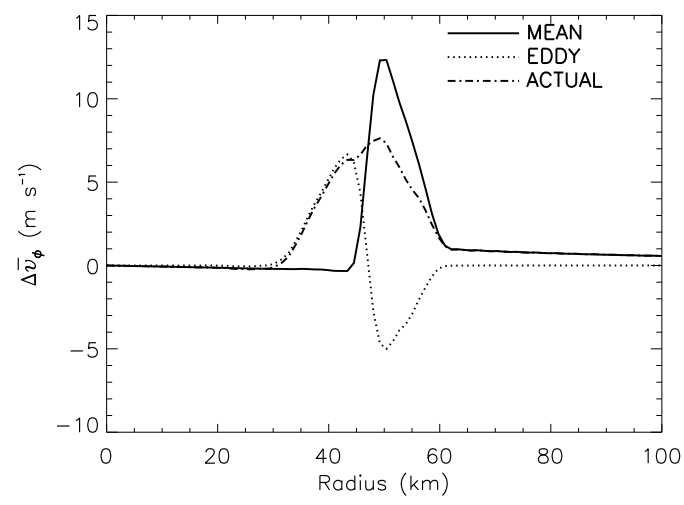

FIG. 11. Effect of Rayleigh friction for thin heating region with $\alpha^{-1}=2.0 \times 10^{4} \mathrm{~s}$. (a) No friction (C1); (b) with friction (C1X), $\mu=4 \times 10^{-6} \mathrm{~s}^{-1}$, corresponding to vorticity half-life of $48 \mathrm{~h}$; (c) tangential momentum budget for no friction case $(\mathrm{C} 1)$; and (d) tangential momentum budget for friction case (C1X). Wind vectors are overlaid on PV plots.

directly specified through a mass sink, and the diabatic heating induces convergence and generates PV. A logistic limiter is not necessary in this modeling framework since the temporal variation of the diabatic heating is directly specified.

The effects of friction were also examined through the tangential momentum budgets. In Figs. $11 \mathrm{c}$ and $11 \mathrm{~d}$, comparisons are made between the tangential momentum budgets of experiments C1 and C1X. In Figs. 11c and $11 \mathrm{~d}$, it is found that Rayleigh friction reduces the mean spinup associated with the eyewall mass sink by approximately $3 \mathrm{~m} \mathrm{~s}^{-1}$. Friction acts to reduce both the negative contribution of the eddy term in the eyewall and the positive contribution in the outer eye. This occurs because friction reduces the PV everywhere, which would reduce the eddy PV flux $\left(\overline{h P^{*} v_{r}^{*}}\right)$. The reduction in the negative contribution of the eddy term in the eyewall has the net effect of increasing the actual spinup in the eyewall as compared to the case of no friction. Also, the peak spinup is at a larger radius (in the eyewall mass-sink region) than in the case of no friction (in the outer eye). Therefore, friction acts to reduce the asymmetric contraction of the radius of maximum winds.

\section{b. Variation in diffusion}

Diffusion is used in the numerical model in order to control spectral blocking associated with potential enstrophy cascade to small scales. In the control experiments, $\nu=25 \mathrm{~m}^{2} \mathrm{~s}^{-1}$, corresponding to a $1 / e$-damping time of $3.5 \mathrm{~h}$ for all Fourier modes having total wavenumber 170. To understand how diffusion affects the PV ring evolution and momentum budgets, a sensitivity test was performed for the thin heating experiment $\mathrm{C} 1$ with increased $\nu=100 \mathrm{~m}^{2} \mathrm{~s}^{-1}$ (corresponding to a $1 / e$-damping time of $0.9 \mathrm{~h}$ ). This experiment is $\mathrm{C} 1 \mathrm{Y}$ in Table 2. A comparison of experiments $\mathrm{C} 1$ and $\mathrm{C} 1 \mathrm{Y}$ is 
a)

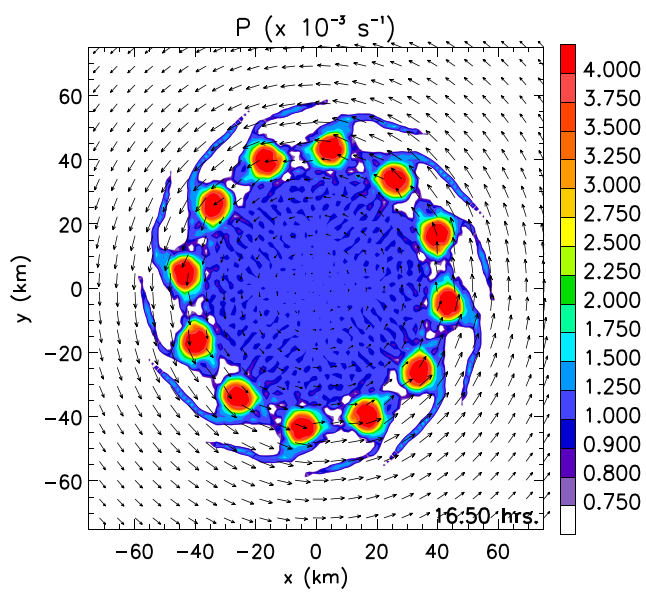

c)

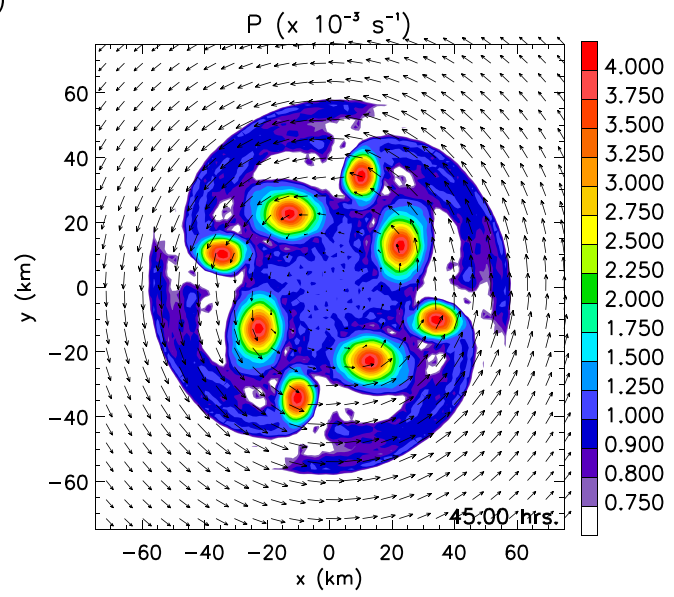

e)

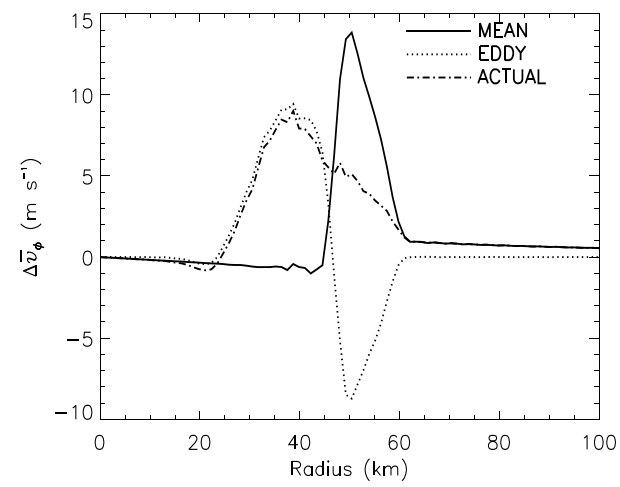

b)

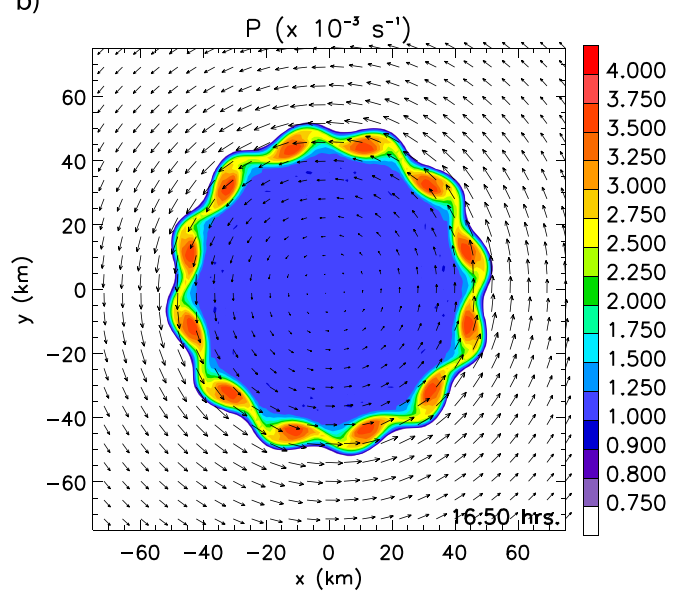

d)

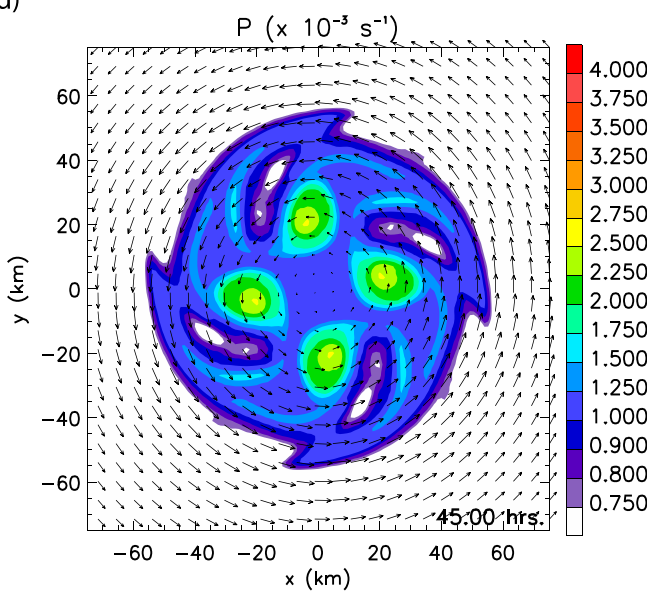

f)

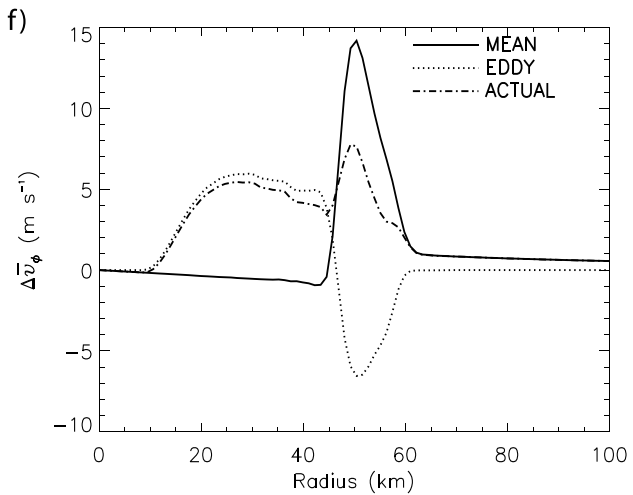

FIG. 12. Effect of diffusion for thin heating region with $\alpha^{-1}=2.0 \times 10^{4} \mathrm{~s}$ : (a) experiment $\mathrm{C} 1$ at $t=15 \mathrm{~h}(\nu=$ $\left.25 \mathrm{~m}^{2} \mathrm{~s}^{-1}\right)$, (b) experiment $\mathrm{C} 1 \mathrm{Y}$ at $t=15 \mathrm{~h}\left(\nu=100 \mathrm{~m}^{2} \mathrm{~s}^{-1}\right)$, (c) experiment $\mathrm{C} 1$ at $t=45 \mathrm{~h}\left(\nu=25 \mathrm{~m}^{2} \mathrm{~s}^{-1}\right)$, (d) experiment $\mathrm{C} 1 \mathrm{Y}$ at $t=45 \mathrm{~h}\left(\nu=100 \mathrm{~m}^{2} \mathrm{~s}^{-1}\right)$, (e) $\mathrm{C} 10-48$-h integrated tangential momentum budget, and (f) $\mathrm{C} 1 \mathrm{Y}$ $0-48$-h integrated tangential momentum budget. Wind vectors are overlaid on PV plots.

given in Fig. 12. In Figs. 12a and 12b, it is evident that increased diffusion causes the PV ring to be slightly more stable; however, the most unstable mode of azimuthal wavenumber 12 is unchanged between experiments. At $t=45 \mathrm{~h}$ (Figs. 12c,d), experiment $\mathrm{C} 1$ has eight mesovortices, while experiment $\mathrm{C} 1 \mathrm{Y}$ has four. In $\mathrm{C} 1 \mathrm{Y}$, mergers occurred earlier causing a reduction in mesovortices. Also note that at $t=45 \mathrm{~h}$, the mesovortices in $\mathrm{C} 1 \mathrm{Y}$ are at a smaller radius. In Figs. 12e and 12f, the tangential momentum budget is shown for each experiment. 
The mean term is not affected by the increased diffusion, which is expected since diffusion is scale selective (higher wavenumbers are damped at higher rates). However, the eddy term is quite different between experiments. As in the case of Rayleigh friction, diffusion damps the PV in eddies, and therefore reduces both the magnitude of the negative contribution in the eyewall and the positive contribution in the outer eye. Additionally, in C1Y, the influence of the eddy term is drawn radially inward from $\mathrm{C} 1$. Overall, variability in diffusion effects details of the eddy processes (i.e., mesovortex evolution), which can have important effects on the eddy term in the tangential momentum budget. Experiments with less eddy activity will thus be much less sensitive to variation in the diffusivity.

\section{c. Variation in transition zones}

In the control experiments, a 4-km-wide smooth transition zone was selected between the eye and eyewall mass sink. A sensitivity test was conducted for the thin ring experiment $\mathrm{C} 1$ with wider $8-\mathrm{km}$ transition zones (experiment C1Z). Since the finest gridpoint resolution is $1.17 \mathrm{~km}$, it is possible that $4 \mathrm{~km}$ is just at the threshold of being able to smoothly represent the masssink function in the discrete model. It is found that the evolution of $\mathrm{C} 1 \mathrm{Z}$ is similar to $\mathrm{C} 1$ in terms of the most unstable mode and nonlinear evolution of the mesovortices. In $\mathrm{C} 1 \mathrm{Z}, \mathrm{PV}$ production in the inner transition zone yields a broader PV distribution. However, the outer 8-km-wide smooth transition zone is eradicated by the radial PV advection, producing a quasi-"top hat" radial PV profile at the outer edge (as in the 4-km transition zone experiment $\mathrm{C} 1$ ). Therefore, the induced radial PV advection causes a steepening of radial PV gradient at the outer edge of the ring, regardless of radial smoothness the heating function.

\section{d. Heating profiles reversed with inner radii constant}

In the control experiments, the outer radius of heating was held fixed, and the inner radius was varied to create the thick, middle, and thin rings. A natural question is how the results would change if the inner radius of the heating was held fixed and the outer radii varied. Two experiments were conducted using $\alpha^{-1}=1.0 \times 10^{4} \mathrm{~s}$ for the middle and thin heating rings, but with the inner radius held fixed (Table 2, experiments $\mathrm{B} 2 \mathrm{X}$ and $\mathrm{C} 2 \mathrm{X}$, respectively). Note that the thick ring experiment $\mathrm{A} 2$ is unchanged since the heating region spans the maximum inner and outer radius. The corresponding mass-sink profiles are given in Fig. 13b, with the original ones shown in Fig. 13a. Since the net heating has to be the same, note that the thin ring must now have a significantly larger mass sink in the new configuration than in the older one. The intensity curves for the experiments of Fig. 13a are given in Fig. 13b, and the intensity curves for the experiments in Fig. 13c are given in Fig. 13d. There is a very large sensitivity to variations in the heating structure. When the outer radius was held fixed, the thin heating ring had the lowest intensity (which was further reduced by the onset of barotropic instability), while the middle ring had a higher intensity, and the thick ring had the highest intensity. Note that both the middle and thick rings remained stable. However, with the inner radius held fixed, the thin heating ring has the largest intensification rate and maximum intensity, followed by the middle and thick rings, respectively. In this configuration, the middle and thick rings remained stable, while the thin ring developed a polygonal asymmetry without a complete breakdown.

These results highlight the importance of the radial distribution of the net heating. If the net heating is distributed into a thin ring near the axis of rotation, rapid intensification to a high peak intensity can occur. This is believed to be caused by three factors: (i) the peak magnitude of the mass sink is increased by having the net heating confined to a small area, making the $P Q$ term in (6) very large; (ii) thin rings at small radii are more prone to be stable according to Schubert et al. (1999) because the thickness parameter $\left[\left(r_{1}+r_{2}\right) /\left(r_{3}+r_{4}\right)\right]$ is smaller, making the barotropic instability and breakdown in mesovortices less likely; and (iii) since the heating is well inside the radius of maximum winds, the induced erosion of the PV at the outer edge is not initially in the region of the heating. This result partially explains how hurricanes with very small eyes can rapidly intensify initially to high peak intensities. These hurricanes have deep convective eyewall heating confined close to the axis of rotation. An observational example of a storm that exhibited this behavior is Hurricane Wilma (2005). The National Hurricane Center tropical cyclone report on Wilma (Pasch et al. 2013) stated the following: "In the span of just 24 hours, Wilma had intensified from a 60-kt tropical storm to a $150-k t$ category 5 hurricane, an unprecedented event for an Atlantic tropical cyclone. It is fortunate that this ultra-rapid strengthening took place over open waters, apparently void of ships, and not just prior to a landfall. Wilma reached its peak sustained wind speed of $160 \mathrm{kt}$ at around 1200 UTC 19 October. During the strengthening episode, Air Force reconnaissance observations indicated that the eye of the hurricane contracted to a diameter of $2 \mathrm{nmi}$; this is the smallest eye known to National Hurricane Center (NHC) staff." Our results suggest a possible connection between rapid intensification and storms with small eyes, as documented by the Wilma case. 
a)

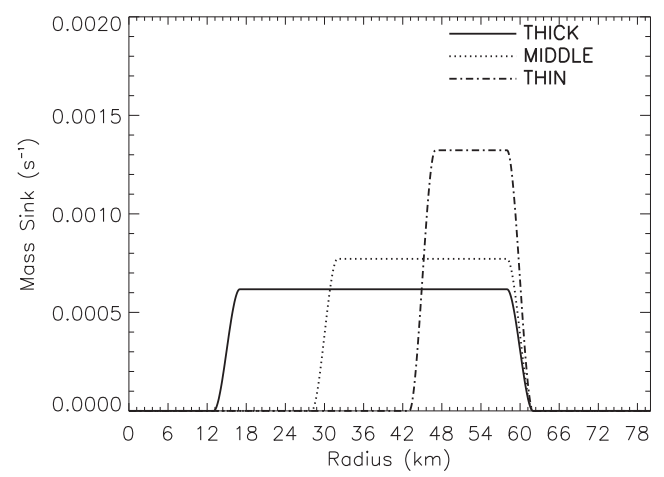

b)

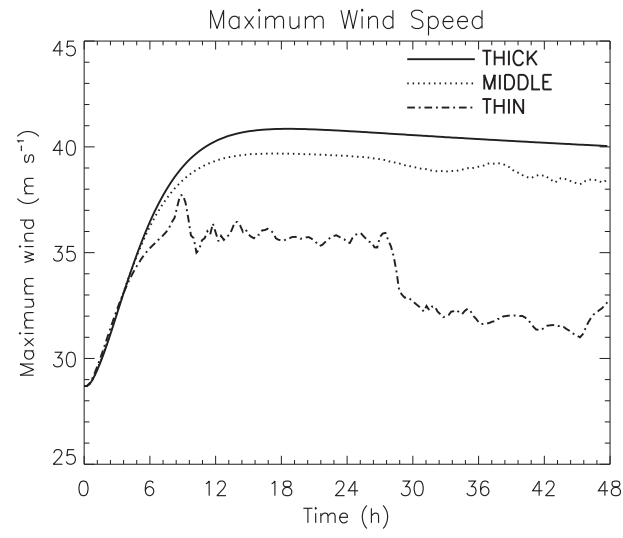

c)

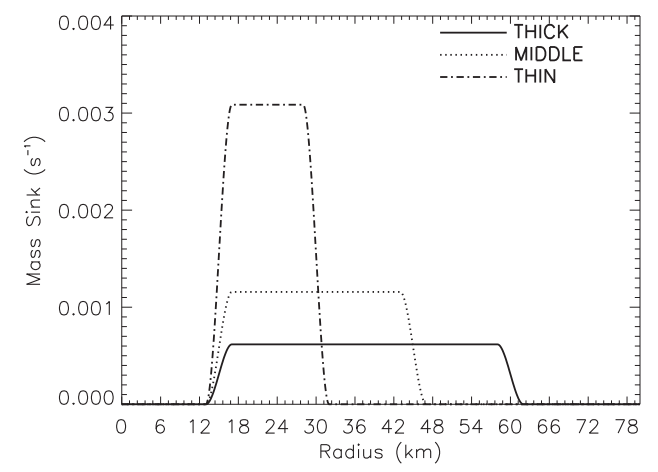

d)

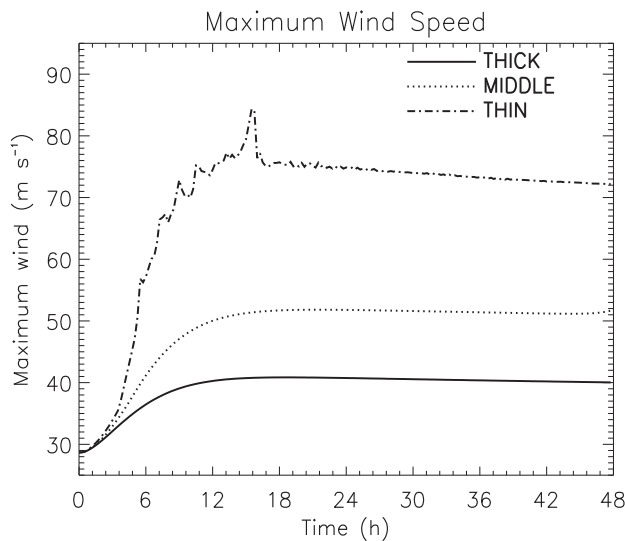

FIG. 13. Reversed mass-sink profiles with $\alpha^{-1}=1.0 \times 10^{4} \mathrm{~s}$. (a) Mass sinks with same outer radius (A2, B2, C2) and (b) corresponding intensity curves; (c) mass sinks with same inner radius (A2, B2X, C2X) and (d) corresponding intensity curves. Note the change in the ordinate scale between (a) and (c), and between (b) and (d).

\section{e. Variation in heating location}

In the next sensitivity test, the heating location is varied for experiment B2, and the results are given in Fig. 14. The initial-vortex relative vorticity and tangential velocity are shown, with the location of the $30-\mathrm{km}$ wide heating region denoted by the red box, which varies from inside the Rankine vortex (B2; Fig. 14a), to the edge (B2Y; Fig. 14b), to the outside (B2Z; Fig. 14c). In Fig. 14d, the corresponding intensity curves versus time are shown for the different heating locations. Heating inside the Rankine vortex produces the most rapid intensification rates and maximum intensity. The intensification rate and maximum intensity drops significantly for the heating at the edge, and there is no intensification for the case of heating outside the edge. These results highlight the critical importance of the location of axisymmetric heating in hurricanes with respect to intensity variability. Consistent with the results of Hack and Schubert (1986), Vigh and Schubert (2009), and Musgrave et al. (2012), heating in the region of high inertial stability inside the radius of maximum winds is effective at spinning up the vortex, while heating in the region of low inertial stability outside the radius of maximum winds does not contribute to vortex intensification. However, the latter heating does increase the size of the vortex, and hence its area-integrated kinetic energy. These heating location results are consistent with the observational study of Rogers et al. (2013), who found that intensifying tropical cyclones had more convective bursts inside the radius of maximum winds, while steady-state tropical cyclones had more convective bursts outside the radius of maximum winds.

\section{f. Variation in Coriolis parameter}

A sensitivity test was done to investigate the effect of variations in the Coriolis parameter for experiment $\mathrm{A} 1$ (Table 2, experiments A1X and A1Y). Experiment A1 (thick heating and $\alpha^{-1}=2.0 \times 10^{4} \mathrm{~s}$ ) was executed with two different values of $f$, corresponding to $5^{\circ} \mathrm{N}(f=$ $\left.1.27 \times 10^{-5} \mathrm{~s}^{-1}\right)(\mathrm{A} 1 \mathrm{X})$ and $25^{\circ} \mathrm{N}\left(f=6.16 \times 10^{-5} \mathrm{~s}^{-1}\right)$ (A1Y). The results of all three experiments are given in Fig. 15. As shown in Fig. 15, the intensity is not very sensitive to variations in the Coriolis parameter. There is 
a) INSIDE

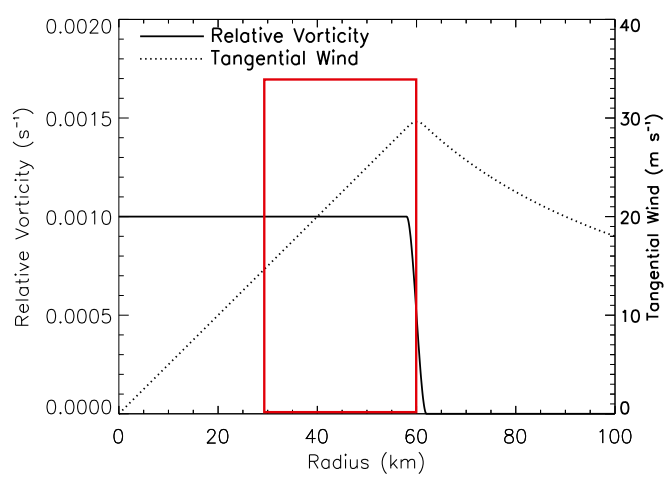

c) OUTSIDE

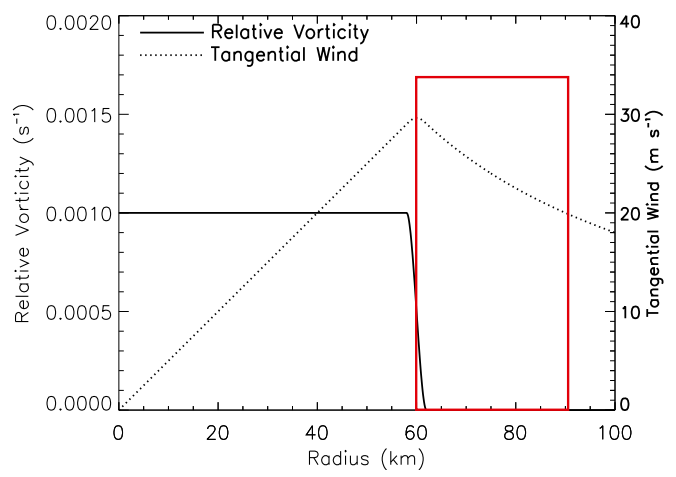

b) EDGE

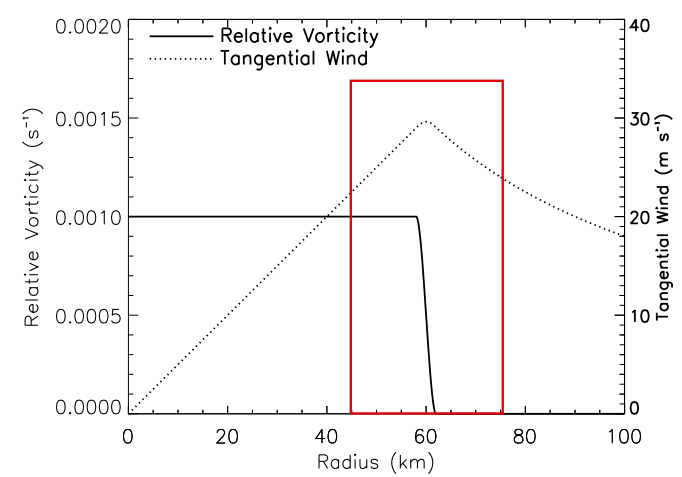

d)

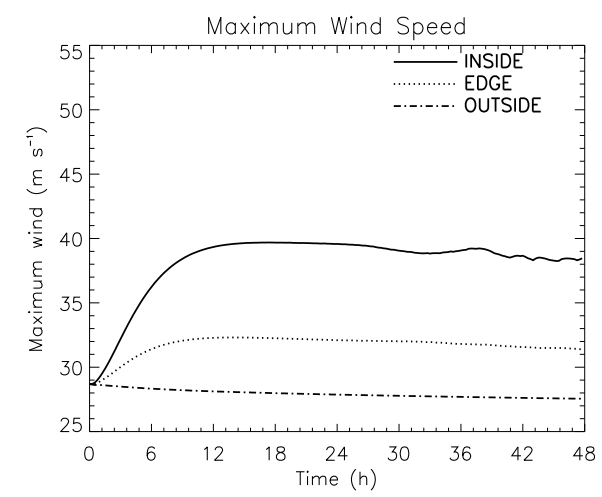

FIG. 14. Variation of the location of heating with $\alpha^{-1}=1.0 \times 10^{4} \mathrm{~s}$ : (a) inside the edge of the Rankine vortex (B2), (b) centered on the edge (B2Y), (c) outside the edge (B2Z), and (d) corresponding intensity curves. The red box marks the $30-\mathrm{km}$-wide location of axisymmetric heating.

virtually no difference in the intensification rate, and only minor differences in the maximum intensity. The simulation at $25^{\circ} \mathrm{N}$ (A1Y) achieves the highest maximum intensity. These results are not surprising since the basic-state relative vorticity is much larger than $f$. These results also indicate that a vortex with a larger basicstate relative vorticity would achieve a higher maximum intensity. In a more realistic full-physics modeling study, $\mathrm{Li}$ et al. (2012) found a stronger sensitivity to the Coriolis parameter, whereby the TC with the lowest value of the Coriolis parameter had the largest intensification rate. Their explanation was that stronger and deeper subgradient inflow developed in this case resulting in larger intensification rates.

\section{g. Heating proportional to the relative vorticity}

A final sensitivity test was conducted with the heating (mass sink) proportional to the relative vorticity, following the parameterization of Wang and Holland (1995),

$$
Q(x, y, t)=\left\{\begin{array}{ll}
\beta \zeta(x, y, t) \alpha^{2} t e^{-\alpha t} & \zeta>0 \\
0 & \zeta \leq 0
\end{array},\right.
$$

where $\beta=0.07$ and $\alpha^{-1}=2.0 \times 10^{4} \mathrm{~s}$. In all of the previous experiments, the heating region was held fixed, so this provides a test to see how the results vary when the heating is proportional to the two-dimensional relative vorticity field (Table 2, experiment D1). The temporal variation is included to the forcing function to mimic a slow episodic heating event.

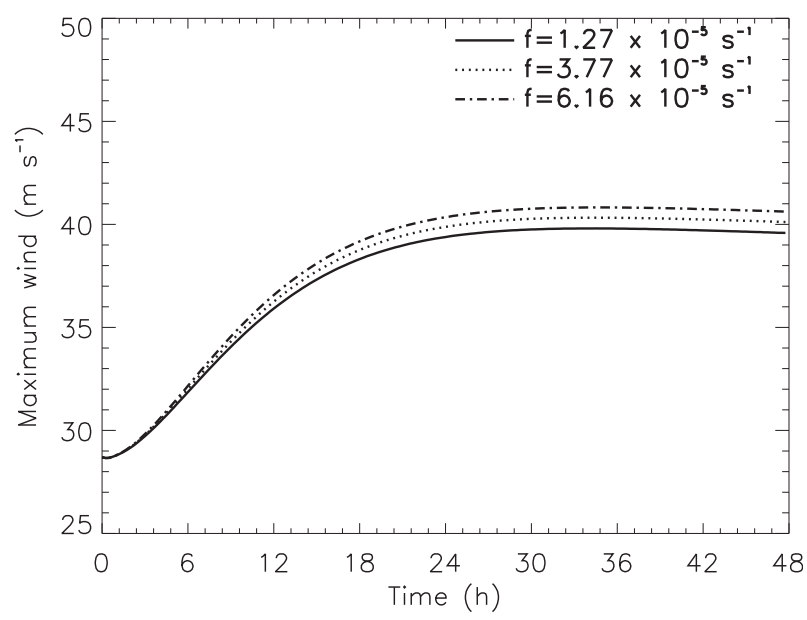

FIG. 15. Intensity curves with varying Coriolis parameter for the thick heating annulus with $\alpha^{-1}=2.0 \times 10^{4} \mathrm{~s}$ for experiments $\mathrm{A} 1 \mathrm{X}\left(f=1.27 \times 10^{-5} \mathrm{~s}^{-1}\right), \mathrm{A} 1\left(f=3.77 \times 10^{-5} \mathrm{~s}^{-1}\right)$, and $\mathrm{A} 1 \mathrm{Y}$ $\left(f=6.16 \times 10^{-5} \mathrm{~s}^{-1}\right)$. 
a)
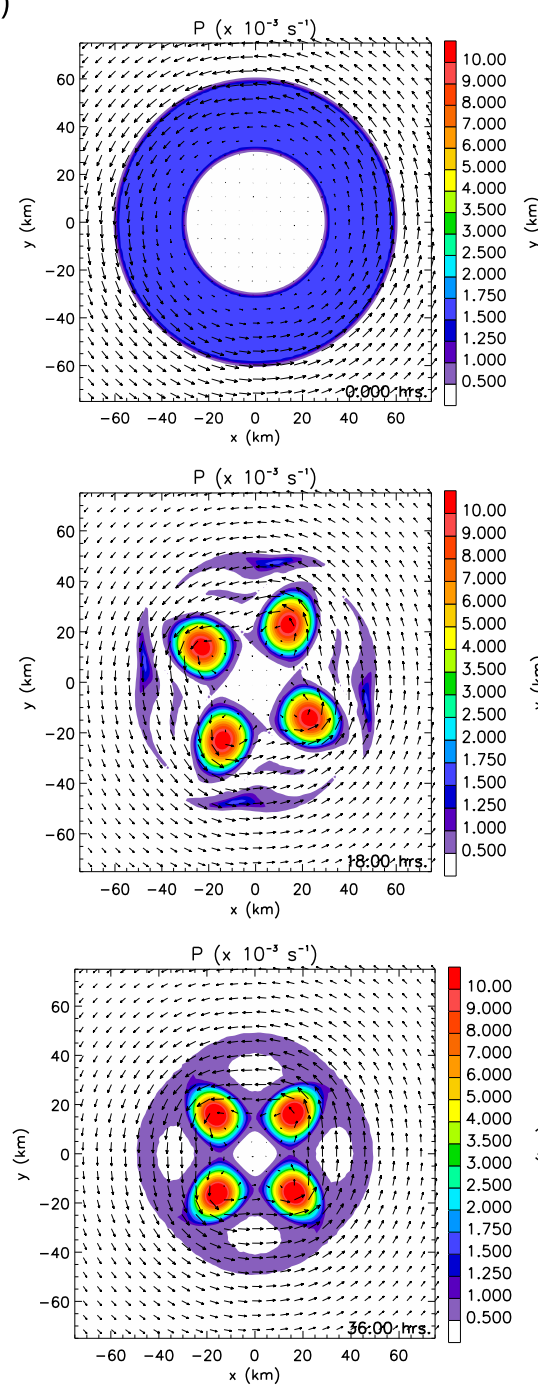

b)

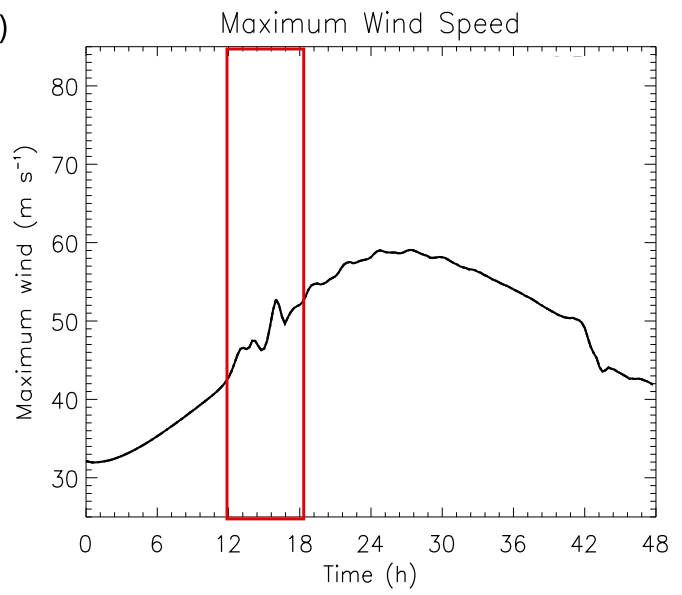

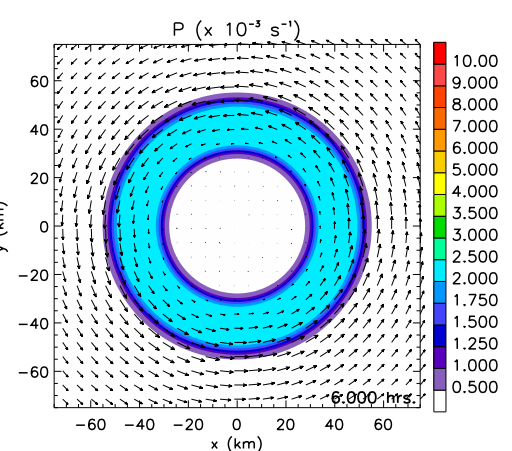
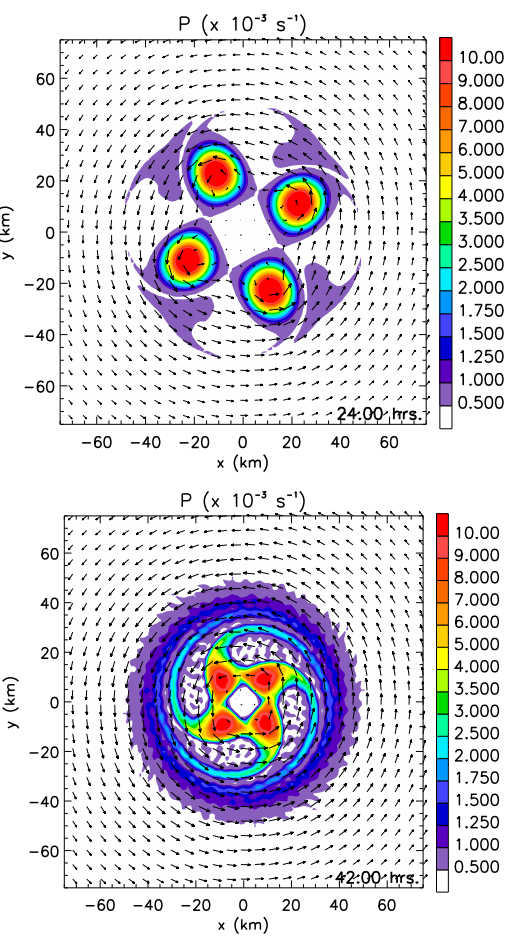
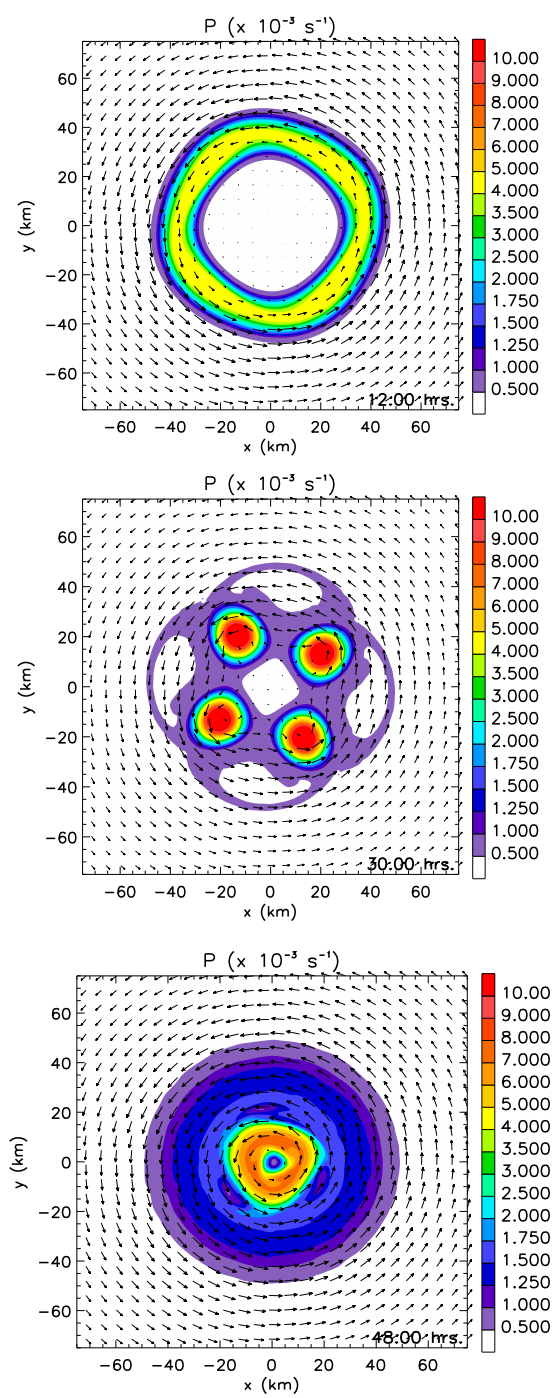

Minimum Pressure

c)

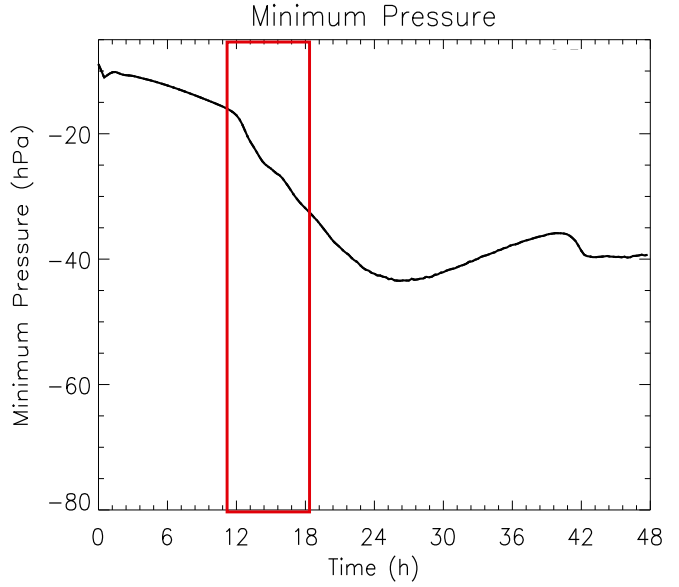

FIG. 16. The experiment D1 with heating proportional to the vorticity. (a) The evolution of the potential vorticity at 6-h increments with wind vectors overlaid, (b) the maximum velocity vs time, and (c) the minimum pressure vs time. The red box denotes the time period of barotropic instability. 
The evolution of experiment D1 is given in Fig. 16a. The initial condition for this experiment is a vorticity ring instead of a Rankine vortex because of the nature of the forcing. Similar to the other experiments, the PV increases and thins in time in response to the mass sink. Barotropic instability sets in at approximately $t=12 \mathrm{~h}$, and the PV ring has broken down into four mesovortices by $t=18 \mathrm{~h}$. At this time, since the heating function is nonzero and near its maximum (Fig. 3), these are convective mesovortices (i.e., mass sinks are located directly over them, helping to further increase their PV). This increase of PV is in the mesovortices is evident in comparing the magnitude of the $\mathrm{PV}$ in the ring at $t=$ $12 \mathrm{~h}$ versus the mesovortices at $t=18 \mathrm{~h}$. As the heating tapers off at $t=30 \mathrm{~h}$ (Fig. 3), the pattern of four mesovortices remain, and finally after some time, there is a merger into a smaller PV ring with a noticeable tripole structure.

In Figs. 16b and 16c, the intensity variability is shown, with the barotropic instability period shown in the red box. In this experiment, the maximum wind increases and the minimum pressure decreases (i.e., the vortex intensifies by maximum wind and minimum pressure) during barotropic instability. This contrasts with previous unforced studies that depict simultaneous decreases in the maximum wind and minimum pressure. This is interesting because the Hendricks et al. (2012) observational study documented a similar intensity evolution during barotropic instability in Hurricane Dolly (2008). There was both a rapid intensification and rapid deepening event coincident with barotropic instability. This experiment suggests that convectively modified barotropic instability, where a PV ring rolls up into convective mesovortices, may be quite different than adiabatic barotropic instability with regard to vortex intensity variability. Nguyen et al. (2011) found that vortical hot towers were initiated as a result of barotropic-convective instability. The deep convective mesovortices here are analogous to the vortical hot towers formed in their fullphysics study.

\section{Conclusions}

The evolution of tropical storm-like vortices under the influence of spatially and temporally varying annular heating has been examined in a shallow-water model, where deep convective heating is parameterized as a mass sink. Annular heating produces a strengthening and thinning PV ring due to the combined effects of the heating and radial PV advection by the induced divergent circulation. The divergent circulation advects low-PV fluid inward, eroding the PV at the outer edge of the heated region. The radial PV advection has two consequences: the radius of maximum winds contracts and the maximum wind magnitude is reduced. Depending on the radial structure, duration, and onset rate of the heating, the PV ring can become thin enough so that the inner and outer counter-propagating vortex Rossby waves interact, leading to barotropic instability and the breakdown of the ring. Similar qualitative results to previous nondivergent studies were obtained, in that thin PV rings tend to break down more rapidly, and at higher azimuthal wavenumbers, than thicker rings. For a broad and weak annular heating region, the forcing may produce a thick ring that can remain stable. For rings that became barotropically unstable, the breakdown led to a transient intensification brake similar to that noted by Rozoff et al. (2009). However, when the heating was prescribed to be proportional to the relative vorticity, the maximum velocity increased and the minimum pressure decreased during barotropic instability, which has been observed in the case of Hurricane Dolly (2008) (Hendricks et al. 2012). This demonstrates that this particular type of forcing may be more applicable to the real atmosphere.

Overall, a significant conclusion from this study is that with a constant net heating, the radial structure, temporal variation, and location of the annular heating region is of critical importance for hurricane intensity variability. This study confirms past results that it is critical to heat in the region of high inertial stability to spin up the hurricane vortex. We also demonstrated that if the net heating is distributed very close to the storm center (or axis or rotation), rapid intensification to a high peak intensity can occur. This result partially explains why tropical cyclones with very small eyes can rapidly intensify to high peak intensities. In closing, we wish to note that the idealized experiments described here are vast oversimplifications of the real atmosphere. In addition to the simplified geometry and forcing structure, the one-layer shallow-water model is not capable of examining the role of vertical shear and other environmental factors in the evolution of hurricane-like vortices. Future work should be focused on three-dimensional idealized experiments in more complicated settings.

Acknowledgments. EH and MP acknowledge the support of the Chief of Naval Research PE-0601153N. WS acknowledges support from NSF Grants AGS-1147120 and AGS-1250966. YC and HK acknowledge the support of the Naval International Cooperative Opportunities in Science and Technology Program Grant N62909-11-17096. We thank Christopher Rozoff, Qingfang Jiang, and Scott Fulton for helpful discussions. This manuscript was improved by the helpful comments of Paul Reasor and two anonymous reviewers. 


\section{REFERENCES}

Andrews, D. G., 1983: A finite-amplitude Eliassen-Palm theorem in isentropic coordinates. J. Atmos. Sci., 40, 1877-1883, doi:10.1175/1520-0469(1983)040<1877:AFAEPT>2.0.CO;2.

_ J. R. Holton, and C. B. Leovy, 1987: Middle Atmosphere Dynamics. Academic Press, 489 pp.

Chen, Y., and M. K. Yau, 2001: Spiral bands in a simulated hurricane. Part I: Vortex Rossby wave verification. J. Atmos. Sci., 58, 2128-2145, doi:10.1175/1520-0469(2001)058<2128: SBIASH $>2.0 . \mathrm{CO} ; 2$.

Guinn, T. A., and W. H. Schubert, 1993: Hurricane spiral bands. J. Atmos. Sci., 50, 3380-3403, doi:10.1175/ 1520-0469(1993)050<3380:HSB $>2.0$. CO 2 .

Hack, J. J., and W. H. Schubert, 1986: Nonlinear response of atmospheric vorticies to heating by organized cumulus convection. J. Atmos. Sci., 43, 1559-1573, doi:10.1175/ 1520-0469(1986)043<1559:NROAVT>2.0.CO;2.

Hendricks, E. A., and W. H. Schubert, 2010: Adiabatic rearrangement of hollow PV towers. J. Adv. Model. Earth Syst., 2 (8), 19 pp., doi:10.3894/JAMES.2010.2.8.

—_ M. T. Montgomery, and C. A. Davis, 2004: The role of "vortical" hot towers in the formation of Tropical Cyclone Diana (1984). J. Atmos. Sci., 61, 1209-1232, doi:10.1175/ 1520-0469(2004)061<1209:TROVHT>2.0.CO;2.

—, W. H. Schubert, R. K. Taft, H. Wang, and J. P. Kossin, 2009: Life cycles of hurricane-like vorticity rings. J. Atmos. Sci., 66, 705-722, doi:10.1175/2008JAS2820.1.

—_, B. D. McNoldy, and W. H. Schubert, 2012: Observed inner-core structural variability in Hurricane Dolly (2008). Mon. Wea. Rev., 140, 4066-4077, doi:10.1175/ MWR-D-12-00018.1.

Kossin, J. P., and M. D. Eastin, 2001: Two distinct regimes in the kinematic and thermodynamic structure of the hurricane eye and eyewall. J. Atmos. Sci., 58, 1079-1090, doi:10.1175/ 1520-0469(2001)058<1079:TDRITK $>2.0$. CO; 2 .

— terns, and rapid pressure falls in hurricane-like vortices. J. Atmos. Sci., 58, 2196-2209, doi:10.1175/1520-0469(2001)058<2196: MPFPAR $>2.0 . \mathrm{CO} ; 2$.

$\longrightarrow$, and 2004: Mesovortices in Hurricane Isabel. Bull. Amer. Meteor. Soc., 85, 151-153, doi:10.1175/BAMS-85-2-151.

— B. D. McNoldy, and W. H. Schubert, 2002: Vortical swirls in hurricane eye clouds. Mon. Wea. Rev., 130, 3144-3149, doi:10.1175/1520-0493(2002)130<3144:VSIHEC >2.0.CO;2.

Kwon, Y., and W. M. Frank, 2005: Dynamic instabilities of simulated hurricane-like vortices and their impacts on the core structure of hurricanes. Part I: Dry experiments. J. Atmos. Sci., 62, 3955-3973, doi:10.1175/JAS3575.1.

— , and - 2008: Dynamic instabilities of simulated hurricanelike vortices and their impacts on the core structure of hurricanes. Part II: Moist experiments. J. Atmos. Sci., 65, 106-122, doi:10.1175/2007JAS2132.1.

Li, T., X. Ge, M. S. Peng, and W. Wang, 2012: Dependence of tropical cyclone intensification on the Coriolis parameter. Trop. Cyclone Res. Rev., 1, 242-253.

Menelaou, K., M. K. Yau, and Y. Martinez, 2013a: Impact of asymmetric dynamical processes on the structure and intensity change of two-dimensional hurricane-like annular vortices. J. Atmos. Sci., 70, 559-582, doi:10.1175/JAS-D-12-0192.1.

,$- \ldots$, and $-2013 \mathrm{~b}$ : On the origin and impact of a polygonal eyewall in the rapid intensification of Hurricane Wilma (2005). J. Atmos. Sci., 70, 3839-3858, doi:10.1175/JAS-D-13-091.1.
Möller, J. D., and M. T. Montgomery, 1999: Vortex Rossby waves and hurricane intensification in a barotropic model. J. Atmos. Sci., 56, 1674-1687, doi:10.1175/1520-0469(1999)056<1674: VRWAHI $>2.0 . \mathrm{CO} ; 2$.

Montgomery, M. T., and R. J. Kallenbach, 1997: A theory for vortex Rossby waves and its application to spiral bands and intensity changes in hurricanes. Quart. J. Roy. Meteor. Soc., 123, 435-465, doi:10.1002/qj.49712353810.

_ Eigenmode structure. J. Atmos. Sci., 54,1868-1885, doi:10.1175/ 1520-0469(1997)054<1868:FWOBVP $>2.0 . C O ; 2$.

— H. D. Snell, and Z. Yang, 2001: Axisymmetric spindown dynamics of hurricane-like vortices. J. Atmos. Sci., 58, 421-435, doi:10.1175/1520-0469(2001)058<0421:ASDOHL > 2.0.CO;2.

_ , V. A. Vladimirov, and P. V. Denissenko, 2002: An experimental study on hurricane mesovortices. J. Fluid Mech., 471, 1-32, doi:10.1017/S0022112002001647.

—, M. E. Nicholls, T. A. Cram, and A. B. Saunders, 2006: A vortical hot tower route to tropical cyclogenesis. J. Atmos. Sci., 63, 355-386, doi:10.1175/JAS3604.1.

Musgrave, K. D., R. K. Taft, J. L. Vigh, B. D. McNoldy, and W. H. Schubert, 2012: Time evolution of the intensity and size of tropical cyclones. J. Adv. Model. Earth Syst., 4, M08001, doi:10.1029/2011MS000104.

Nguyen, C. M., M. J. Reeder, N. E. Davidson, R. K. Smith, and M. T. Montgomery, 2011: Inner-core vacillation cycles during the intensification of Hurricane Katrina. Quart. J. Roy. Meteor. Soc., 137, 829-844, doi:10.1002/qj.823.

Nguyen, S. V., R. K. Smith, and M. T. Montgomery, 2008: Tropical cyclone intensification and predictability in three dimensions. Quart. J. Roy. Meteor. Soc., 134, 563-582, doi:10.1002/qj.235.

Nolan, D. S., and M. T. Montgomery, 2000: The algebraic growth of wavenumber one disturbances in hurricane-like vortices. J. Atmos. Sci., 57, 3514-3538, doi:10.1175/ 1520-0469(2000)057<3514:TAGOWO >2.0.CO;2.

$\longrightarrow$, and — 2002: Nonhydrostatic, three-dimensional perturbations to balanced, hurricane-like vortices. Part I: Linearized formulation, stability, and evolution. J. Atmos. Sci., 59, 2989-3020, doi:10.1175/1520-0469(2002)059<2989: NTDPTB $>2.0 . \mathrm{CO} ; 2$.

Pasch, R. J., E. S. Blake, H. D. Cobb, and D. P. Roberts, 2013: Hurricane Wilma. Tech. Rep., NWS/NHC Tropical Cyclone Rep. TCR-AL252005, 27 pp. [Available online at http:// www.nhc.noaa.gov/2005atlan.shtml.]

Reasor, P. D., M. T. Montgomery, F. D. Marks, and J. F. Gamache, 2000: Low-wavenumber structure and evolution of the hurricane inner core observed by airborne dual-Doppler radar. Mon. Wea. Rev., 128, 1653-1680, doi:10.1175/1520-0493(2000)128<1653: LWSAEO $>2.0 . \mathrm{CO} ; 2$.

Rodgers, E. B., W. S. Olson, V. M. Karyampudi, and H. F. Pierce, 1998: Satellite-derived latent heating distribution and environmental influences in Hurricane Opal (1995). Mon. Wea. Rev., 126, 1229-1247, doi:10.1175/1520-0493(1998)126<1229: SDLHDA $>2.0 . \mathrm{CO} ; 2$.

Rogers, R., P. D. Reasor, and S. Lorsolo, 2013: Airborne Doppler observations of the inner-core structural differences between intensifying and steady-state tropical cyclones. Mon. Wea. Rev., 141, 2970-2991, doi:10.1175/MWR-D-12-00357.1.

Rozoff, C. M., W. H. Schubert, B. D. McNoldy, and J. P. Kossin, 2006: Rapid filamentation zones in intense tropical cyclones. J. Atmos. Sci., 63, 325-340, doi:10.1175/JAS3595.1.

_ J. P. Kossin, W. H. Schubert, and P. J. Mulero, 2009: Internal control of hurricane intensity: The dual nature of potential 
vorticity mixing. J. Atmos. Sci., 66, 133-147, doi:10.1175/ 2008JAS2717.1.

Schubert, W. H., M. T. Montgomery, R. K. Taft, T. A. Guinn, S. R. Fulton, J. P. Kossin, and J. P. Edwards, 1999: Polygonal eyewalls, asymmetric eye contraction, and potential vorticity mixing in hurricanes. J. Atmos. Sci., 56, 1197-1223, doi:10.1175/ 1520-0469(1999)056<1197:PEAECA > 2.0.CO;2.

Schumacher, C., R. A. Houze, and I. Kraucunas, 2004: The tropical dynamical response to latent heating estimates derived from the TRMM precipitation radar. J. Atmos. Sci., 61, 1341-1358, doi:10.1175/1520-0469(2004)061<1341:TTDRTL > 2.0.CO;2.

Terwey, W. D., and M. T. Montgomery, 2002: Wavenumber-2 and wavenumber- $m$ vortex Rossby wave instabilities in a generalized three-region model. J. Atmos. Sci., 59, 2421-2427, doi:10.1175/1520-0469(2002)059<2421:WAWMVR>2.0.CO;2.

Tung, K. K., 1986: Nongeostrophic theory of zonally averaged circulation. Part I: Formulation. J. Atmos. Sci., 43, 2600-2618, doi:10.1175/1520-0469(1986)043<2600:NTOZAC > 2.0.CO;2.

Vigh, J., and W. H. Schubert, 2009: Rapid development of the tropical cyclone warm core. J. Atmos. Sci., 66, 3335-3350, doi:10.1175/2009JAS3092.1.
Wang, Y., and G. J. Holland, 1995: On the interaction of tropicalcyclone scale vortices. IV: Baroclinic vortices. Quart. J. Roy. Meteor. Soc., 121, 95-126, doi:10.1002/qj.49712152106.

Willoughby, H. E., J. A. Clos, and M. G. Shoreibah, 1982: Concentric eye walls, secondary wind maxima, and the evolution of the hurricane vortex. J. Atmos. Sci., 39, 395-411, doi:10.1175/ 1520-0469(1982)039<0395:CEWSWM > 2.0.CO;2.

Wu, C.-C., H.-J. Cheng, Y. Wang, and K.-H. Chou, 2009: A numerical investigation of the eyewall evolution of a landfalling typhoon. Mon. Wea. Rev., 137, 21-40, doi:10.1175/ 2008MWR2516.1.

Yang, B., Y. Wang, and B. Wang, 2007: The effect of internally generated inner-core asymmetries on tropical cyclone potential intensity. J. Atmos. Sci., 64, 1165-1188, doi:10.1175/ JAS3971.1.

Yau, M. K., Y. Liu, D.-L. Zhang, and Y. Chen, 2004: A multiscale numerical study of Hurricane Andrew (1992). Part VI: Smallscale inner-core structures and wind streaks. Mon. Wea. Rev., 132, 1410-1433, doi:10.1175/1520-0493(2004)132<1410: AMNSOH $>2.0 . \mathrm{CO} ; 2$. 